Supporting Information for

\title{
Electrochemical Detection of Transient Cobalt Hydride Intermediates of Electrocatalytic Hydrogen Production
}

\author{
Eric S. Wiedner* and R. Morris Bullock \\ Center for Molecular Electrocatalysis, Pacific Northwest National Laboratory, \\ P.O. Box 999, K2-57, Richland, Washington 99352 \\ * E-mail: eric.wiedner@pnnl.gov
}

Table of Contents

Page

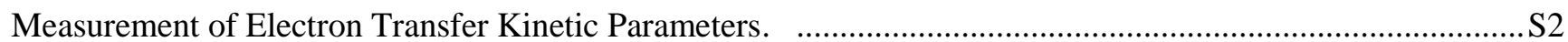

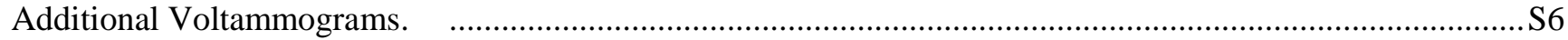

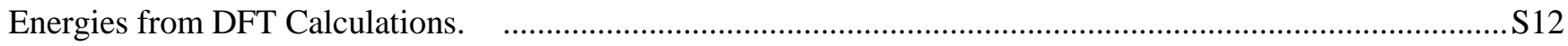

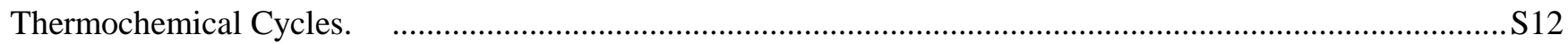

Correlation of Reduction Potentials and Heterolytic/Homolytic Co-H Bond Strengths. .............................S15

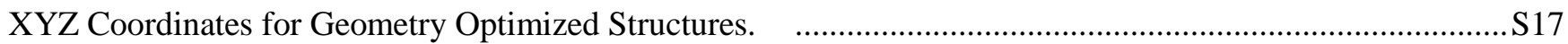




\section{Procedure for Determination of Electron Transfer Kinetic Parameters}

As described in the main text, the cathodic peak potential $\left(E_{\mathrm{pc}}\right)$ and anodic peak potential $\left(E_{\mathrm{pa}}\right)$ for a quasireversible wave are governed by the following equations:

$$
\begin{aligned}
& E_{p c}=E^{\circ \prime}-\frac{0.78 R T}{\alpha F}+\frac{2.303 R T}{\alpha F} \log \left(k_{S} \sqrt{\frac{R T}{\alpha F v D}}\right) \\
& E_{p a}=E^{\circ \prime}+\frac{0.78 R T}{\alpha F}-\frac{2.303 R T}{(1-\alpha) F} \log \left(k_{S} \sqrt{\frac{R T}{(1-\alpha) F v D}}\right)
\end{aligned}
$$

These equations can be rearranged to describe the line obtained from plots of $E_{\mathrm{pc}}$ and $E_{\mathrm{pa}}$ versus $\log (v)$ :

$$
\begin{aligned}
& E_{p c}=-\frac{2.303 R T}{2 \alpha F} \log (v)+\left[E^{\circ \prime}-\frac{0.78 R T}{\alpha F}+\frac{2.303 R T}{\alpha F} \log \left(k_{S} \sqrt{\frac{R T}{\alpha F D}}\right)\right] \\
& E_{p a}=\frac{2.303 R T}{2(1-\alpha) F} \log (v)+\left[E^{\circ \prime}+\frac{0.78 R T}{\alpha F}-\frac{2.303 R T}{(1-\alpha) F} \log \left(k_{S} \sqrt{\frac{R T}{(1-\alpha) F D}}\right)\right]
\end{aligned}
$$

The transfer coefficient $(\alpha)$ can be determined from the slope of a plot of $E_{\mathrm{pc}}$ or $E_{\mathrm{pa}}$ versus $\log (v)$. If the formal potential $\left(E^{\circ \prime}\right)$ and diffusion coefficient $(D)$ are also known, then the standard heterogeneous electron transfer rate constant $\left(k_{\mathrm{S}}\right)$ can be determined from the intercept $(b)$ of a plot of $E_{\mathrm{pc}}$ or $E_{\mathrm{pa}}$ versus $\log (\mathrm{v})$.

$$
\begin{aligned}
& \text { For } E_{p c}: \log \left(k_{S}\right)=\frac{\alpha F}{2.303 R T}\left(b-E^{\circ \prime}\right)+0.339-\log \left(\sqrt{\frac{R T}{\alpha F D}}\right) \\
& \text { For } E_{p a}: \log \left(k_{S}\right)=\frac{(1-\alpha) F}{2.303 R T}\left(E^{\circ \prime}-b\right)+\frac{0.339(1-\alpha)}{\alpha}-\log \left(\sqrt{\frac{R T}{(1-\alpha) F D}}\right)
\end{aligned}
$$




\section{Diffusion Coefficient Measurement.}

A solution of $\left[\mathrm{Co}\left(\mathrm{P}^{\mathrm{IB}}{ }_{2} \mathrm{~N}^{\mathrm{Ph}}\right)\left(\mathrm{CH}_{3} \mathrm{CN}\right)_{3}\right]^{2+}(1.0 \mathrm{mM})$ and ferrocene $(1.0 \mathrm{mM})$ in acetonitrile $\left(0.2 \mathrm{M} \mathrm{NBu}_{4} \mathrm{PF}_{6}\right)$ was prepared by serial dilution, and a series of cyclic voltammograms were recorded with $v=0.1-1.0 \mathrm{~V} \mathrm{~s}^{-1}$. The peak current $\left(i_{\mathrm{p}}\right)$ of each analyte is related to its diffusion coefficient by the the Randles-Sevcik equation:

$$
i_{\mathrm{p}}=0.446 F A[\mathrm{Cat}]_{T}(D v)^{1 / 2}(F / R T)^{1 / 2}
$$

Therefore the ratio of the peak currents for ferrocene and $\left[\mathrm{Co}^{\mathrm{II}}\left(\mathrm{P}^{\mathrm{tBu}}{ }_{2} \mathrm{~N}^{\mathrm{Ph}}\right)\left(\mathrm{CH}_{3} \mathrm{CN}\right)_{3}\right]^{2+}$ are equal to the ratio of the square root of their diffusion coefficients:

$$
\frac{-i_{p a}\left(F c^{+/ 0}\right)}{i_{p c}\left(C o^{I I / I}\right)}=\frac{D_{F c}^{1 / 2}}{D_{C o}^{1 / 2}}
$$

Using the averaged peak current ratio of 1.708(6) obtained over $v=0.1-1.0 \mathrm{~V} \mathrm{~s}^{-1}$ and the diffusion coefficient of $2.4 \mathrm{~cm}^{2} \mathrm{~s}^{-1}$ for ferrocene in acetonitrile (Kadish, Ding, and Milinski, Anal. Chem. 1984, 56, 1741), a diffusion coefficient of $8.2 \times 10^{-6} \mathrm{~cm}^{2} \mathrm{~s}^{-1}$ was measured for $\left[\mathrm{Co}^{\mathrm{II}}\left(\mathrm{P}^{t \mathrm{Bu}}{ }_{2} \mathrm{~N}^{\mathrm{Ph}}{ }_{2}\right)\left(\mathrm{CH}_{3} \mathrm{CN}\right)_{3}\right]^{2+}$.

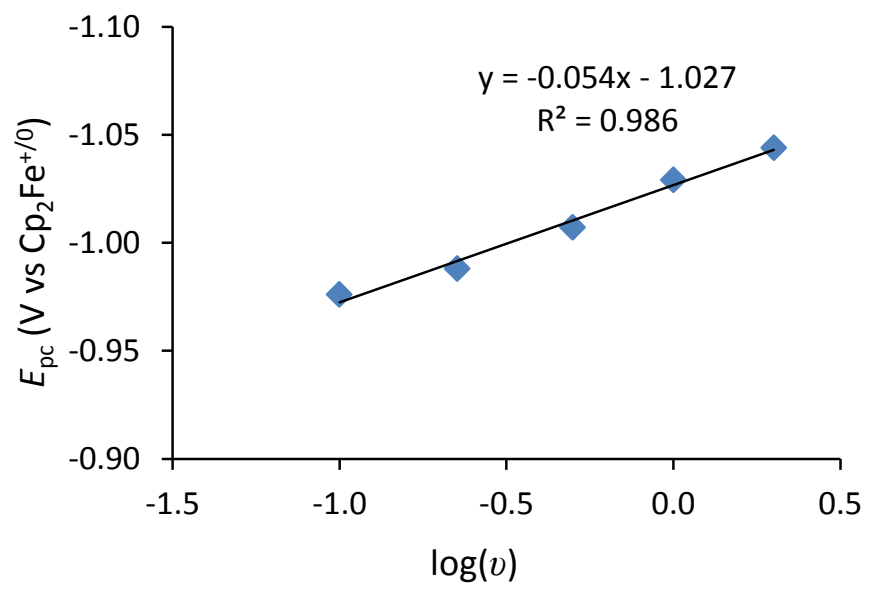

Figure S1. Plot of $E_{\mathrm{pc}}$ versus $\log (v)$ for reduction of $\left[\mathrm{Co}^{\mathrm{II}}\left(\mathrm{P}^{t \mathrm{Bu}}{ }_{2} \mathrm{~N}^{\mathrm{Ph}}{ }_{2}\right)\left(\mathrm{CH}_{3} \mathrm{CN}\right)_{3}\right]^{2+}(1 \mathrm{mM})$ in the presence of p-bromoanilinium tetrafluoroborate $(4 \mathrm{mM})$. Conditions: $0.2 \mathrm{M} \mathrm{NBu}_{4} \mathrm{BF}_{4}$ acetonitrile solution, $1 \mathrm{~mm}$ diameter glassy carbon working electrode. 


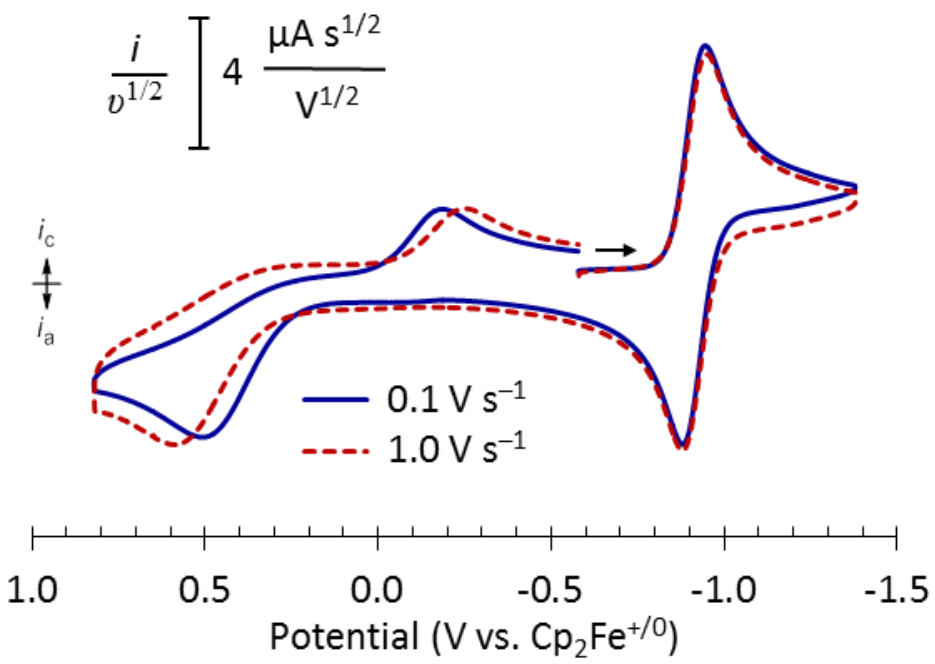

Figure S2. Cyclic voltammograms of $\mathrm{Co}^{\mathrm{II}}\left(\mathrm{dmgBF}_{2}\right)\left(\mathrm{CH}_{3} \mathrm{CN}\right)_{2}$ at varying scan rates. The current has been normalized to $v^{-1 / 2}$. Conditions: $1 \mathrm{mM}$ Co in $0.2 \mathrm{M} \mathrm{NBu}_{4} \mathrm{BF}_{4}$ acetonitrile, $1 \mathrm{~mm}$ diameter glassy carbon working electrode.

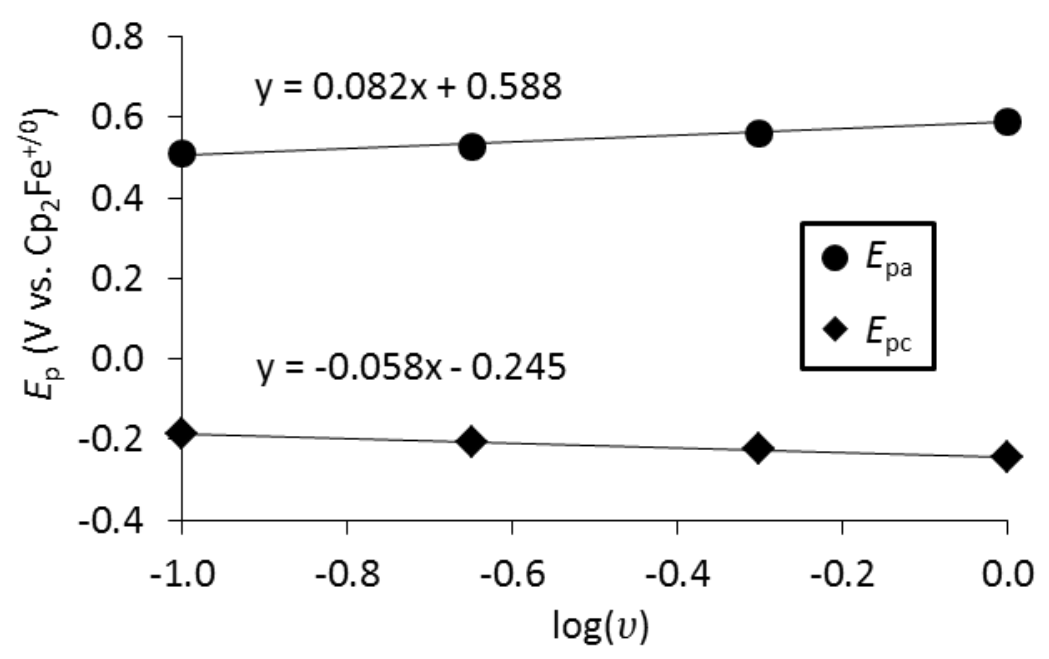

Figure S3. Plot of $E_{\mathrm{p}}$ versus $\log (v)$ for the $\mathrm{Co}^{\mathrm{III} / \mathrm{II}}$ couple of $\mathrm{Co}^{\mathrm{II}}\left(\mathrm{dmgBF}_{2}\right)_{2}\left(\mathrm{CH}_{3} \mathrm{CN}\right)_{2}(1 \mathrm{mM})$. Conditions: $0.2 \mathrm{M}$ $\mathrm{NBu}_{4} \mathrm{BF}_{4}$ acetonitrile solution, $1 \mathrm{~mm}$ diameter glassy carbon working electrode. 


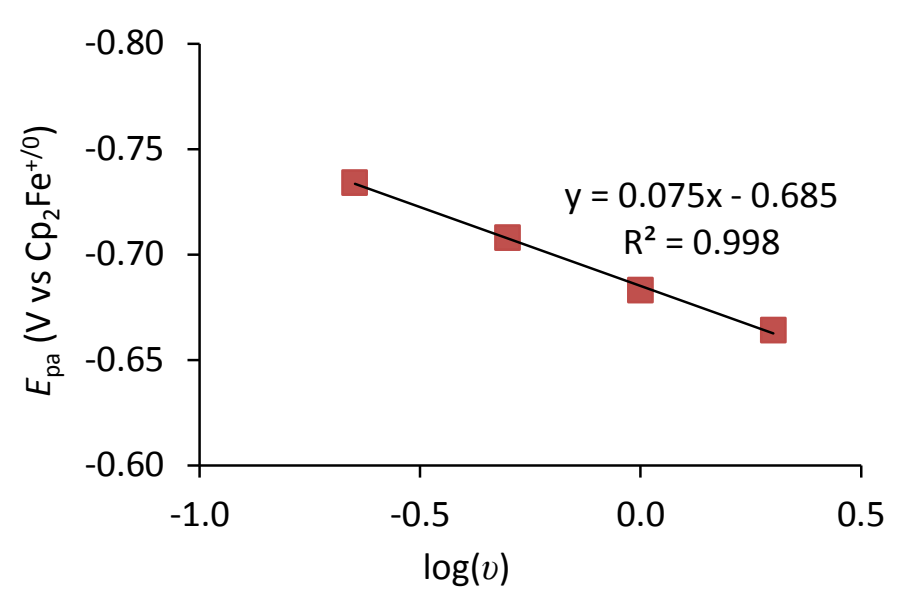

Figure S4. Plot of $E_{\mathrm{pa}}$ versus $\log (v)$ for the anodic wave in voltammograms of $\mathrm{Co}^{\mathrm{II}}\left(\mathrm{dmgBF}_{2}\right)_{2}\left(\mathrm{CH}_{3} \mathrm{CN}\right)_{2}(1 \mathrm{mM})$ in the presence of anilinium tetrafluoroborate $(4 \mathrm{mM})$. Conditions: $0.2 \mathrm{M} \mathrm{NBu}_{4} \mathrm{BF}_{4}$ acetonitrile solution, $1 \mathrm{~mm}$ diameter glassy carbon working electrode.

Table S1. Tabulated Electron Transfer Kinetic Parameters Obtained from Plots of $E_{\mathrm{p}}$ versus $\log (v)$.

\begin{tabular}{|c|c|c|c|c|c|c|c|}
\hline Complex & Couple & Peak & Slope & Intercept & $E^{\circ \prime}(\mathbf{V})$ & $\alpha$ & $k_{\mathrm{S}}\left(\mathrm{cm}^{2} \mathrm{~s}^{-1}\right)$ \\
\hline$\left[\mathrm{Co}^{\mathrm{II}}\left(\mathrm{P}^{t \mathrm{Bu}}{ }_{2} \mathrm{~N}_{2}{ }_{2}\right)\left(\mathrm{CH}_{3} \mathrm{CN}\right)_{3}\right]^{2+}$ & $\mathrm{Co}^{\mathrm{IIITI}}$ & $E_{\mathrm{pc}}$ & -0.057 & 0.211 & 0.33 & 0.52 & $2.5 \times 10^{-3}$ \\
\hline$\left[\mathrm{Co}^{\mathrm{II}}\left(\mathrm{P}^{t \mathrm{Bu}}{ }_{2} \mathrm{~N}_{2}{ }_{2}\right)\left(\mathrm{CH}_{3} \mathrm{CN}\right)_{3}\right]^{2+}$ & $\mathrm{Co}^{\mathrm{III/III}}$ & $E_{\mathrm{pa}}$ & 0.057 & 0.449 & 0.33 & 0.48 & $2.7 \times 10^{-3}$ \\
\hline$\left[\mathrm{Co}^{\mathrm{II}}\left(\mathrm{P}^{t \mathrm{Bu}}{ }_{2} \mathrm{~N}^{\mathrm{Ph}}\right)\left(\mathrm{CH}_{3} \mathrm{CN}\right)_{3}\right]^{2+}$ & $\mathrm{Co}^{\mathrm{III/I}} \mathrm{H}$ & $E_{\mathrm{pc}}$ & -0.054 & -1.027 & $-0.80^{a}$ & 0.55 & $2.3 \times 10^{-4}$ \\
\hline $\mathrm{Co}\left(\mathrm{dmgBF}_{2}\right)_{2}\left(\mathrm{CH}_{3} \mathrm{CN}\right)_{2}{ }^{b}$ & $\mathrm{Co}^{\mathrm{III/II}}$ & $E_{\mathrm{pc}}$ & -0.058 & -0.245 & 0.14 & 0.51 & $1.3 \times 10^{-5}$ \\
\hline $\mathrm{Co}^{\mathrm{II}}\left(\mathrm{dmgBF}_{2}\right)_{2}\left(\mathrm{CH}_{3} \mathrm{CN}\right)_{2}{ }^{b}$ & $\mathrm{Co}^{\mathrm{III/II}}$ & $E_{\mathrm{pa}}$ & 0.082 & 0.588 & 0.14 & 0.64 & $3.1 \times 10^{-5}$ \\
\hline $\mathrm{Co}^{\mathrm{II}}\left(\mathrm{dmgBF}_{2}\right)_{2}\left(\mathrm{CH}_{3} \mathrm{CN}\right)_{2}{ }^{b}$ & $\mathrm{Co}^{\mathrm{III/I}} \mathrm{H}$ & $E_{\mathrm{pa}}$ & 0.075 & -0.685 & -0.75 & 0.61 & $6.8 \times 10^{-3}$ \\
\hline
\end{tabular}

${ }^{a}$ Calculated by DFT. ${ }^{b}$ A diffusion coefficient of $8 \times 10^{-6} \mathrm{~cm}^{2} \mathrm{~s}^{-1}$ was previously reported (Hu, X. L.; Brunschwig, B. S.; Peters, J. C. J. Am. Chem. Soc. 2007, 129, 8988-8998).

For the $\mathrm{Co}^{\text {III/II }}$ couple of $\mathrm{Co}^{\mathrm{II}}\left(\mathrm{dmgBF}_{2}\right)_{2}\left(\mathrm{CH}_{3} \mathrm{CN}\right)_{2}$, very different values of $\alpha$ are obtained from a plot of $E_{\mathrm{pa}}$ versus $\log (v)$ than from $E_{\mathrm{pc}}$ versus $\log (v)(\alpha=0.64$ vs $\alpha=0.51)$. This indicates that the Co ${ }^{\mathrm{III} / \mathrm{II}}$ couple is not a simple oneelectron transfer process. A likely electrochemical oxidation mechanism is a $\mathrm{C}_{\mathrm{r}} \mathrm{E}_{\mathrm{q}}$ mechanism, where a 5-coordinate $\mathrm{Co}^{\mathrm{II}}$ species is in equilibrium with a 6-coordinate $\mathrm{Co}^{\mathrm{II}}$ species, which can then be oxidized to 6-coordinate $\mathrm{Co}^{\mathrm{III}}$ :

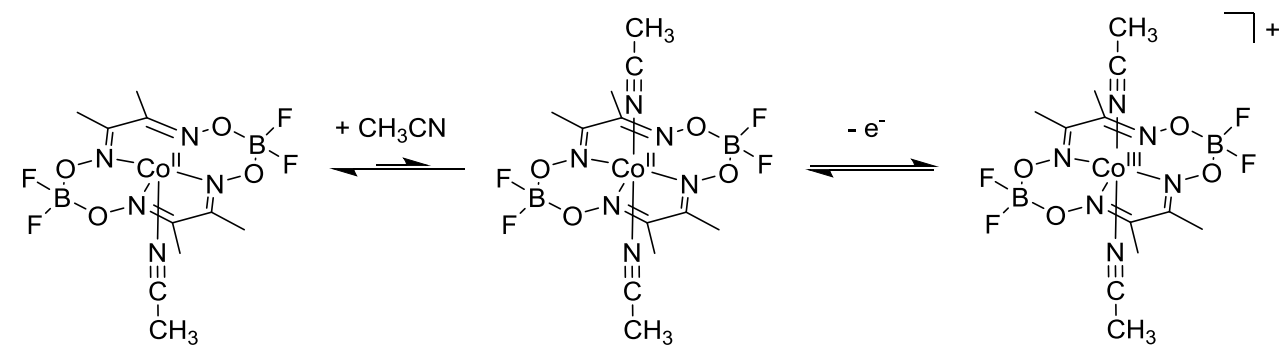

Full quantification of this process would require additional data that is currently unknown, such as the equilibrium constant and kinetics for acetonitrile binding and loss at $\mathrm{Co}$. . Since we have not explicitly accounted for the solvent equilibrium for any of the $\mathrm{Co}^{\mathrm{III} / \mathrm{II}}$ or $\mathrm{Co}{ }^{\mathrm{III} / \mathrm{II}} \mathrm{H}$ couples studied here, the kinetics of solvent binding and dissociation at $\mathrm{Co}^{\mathrm{II}}$ are folded into measured $\alpha$ and $k_{\mathrm{S}}$ values. 


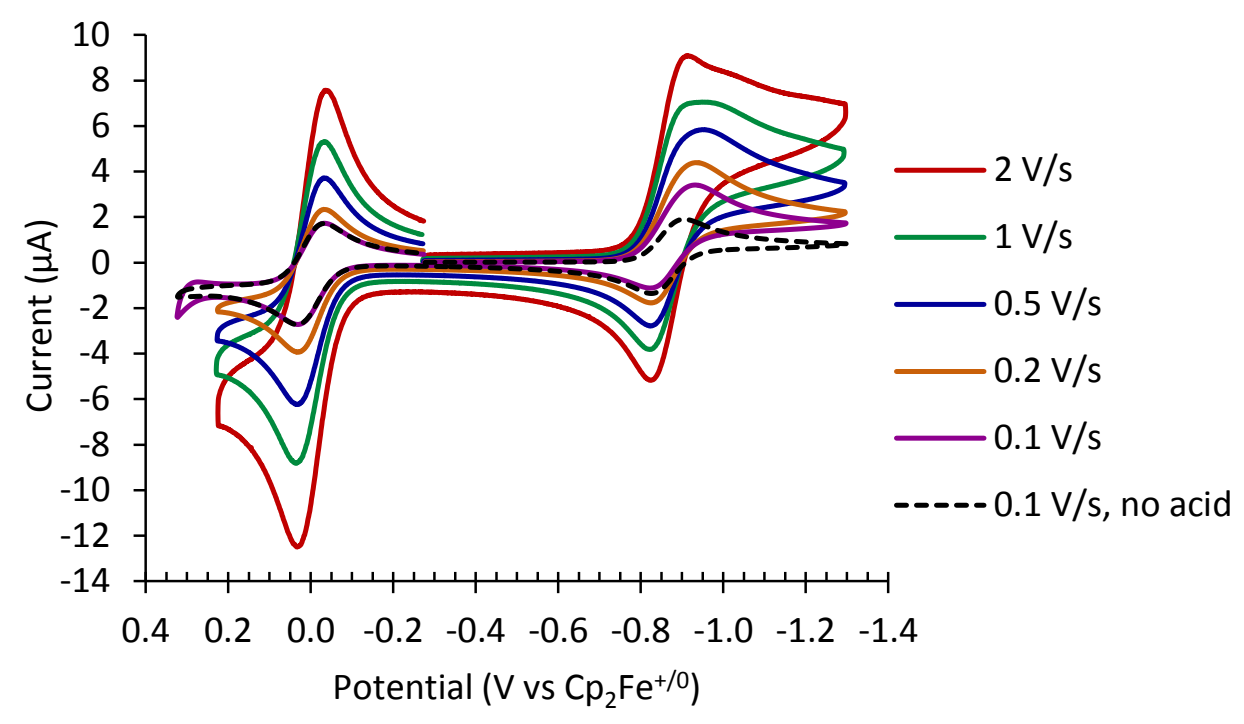

Figure S5. Cyclic voltammograms of $\left[\mathrm{Co}^{\mathrm{II}}\left(\mathrm{P}^{t \mathrm{Bu}}{ }_{2} \mathrm{~N}^{\mathrm{Ph}}{ }_{2}\right)\left(\mathrm{CH}_{3} \mathrm{CN}\right)_{3}\right]^{2+}(1 \quad \mathrm{mM})$ and $p$-bromoanilinium tetrafluoroborate $(1 \mathrm{mM})$ at varying scan rates. Conditions: $0.2 \mathrm{M} \mathrm{NBu}_{4} \mathrm{BF}_{4}$ acetonitrile solution, $1 \mathrm{~mm}$ dia. glassy carbon working electrode.

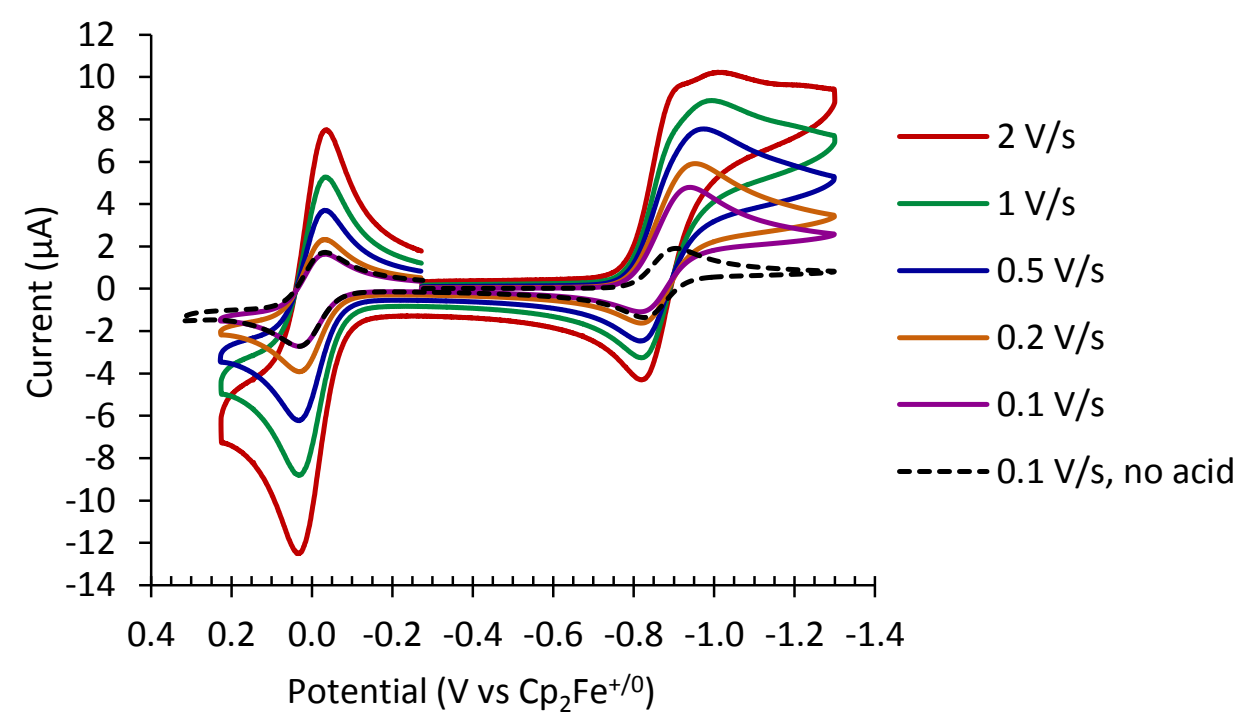

Figure S6. Cyclic voltammograms of $\left[\mathrm{Co}^{\mathrm{II}}\left(\mathrm{P}^{\mathrm{tBu}}{ }_{2} \mathrm{~N}^{\mathrm{Ph}}{ }_{2}\right)\left(\mathrm{CH}_{3} \mathrm{CN}\right)_{3}\right]^{2+}(1 \quad \mathrm{mM})$ and $p$-bromoanilinium tetrafluoroborate $(2 \mathrm{mM})$ at varying scan rates. Conditions: $0.2 \mathrm{M} \mathrm{NBu}_{4} \mathrm{BF}_{4}$ acetonitrile solution, $1 \mathrm{~mm}$ dia. glassy carbon working electrode. 


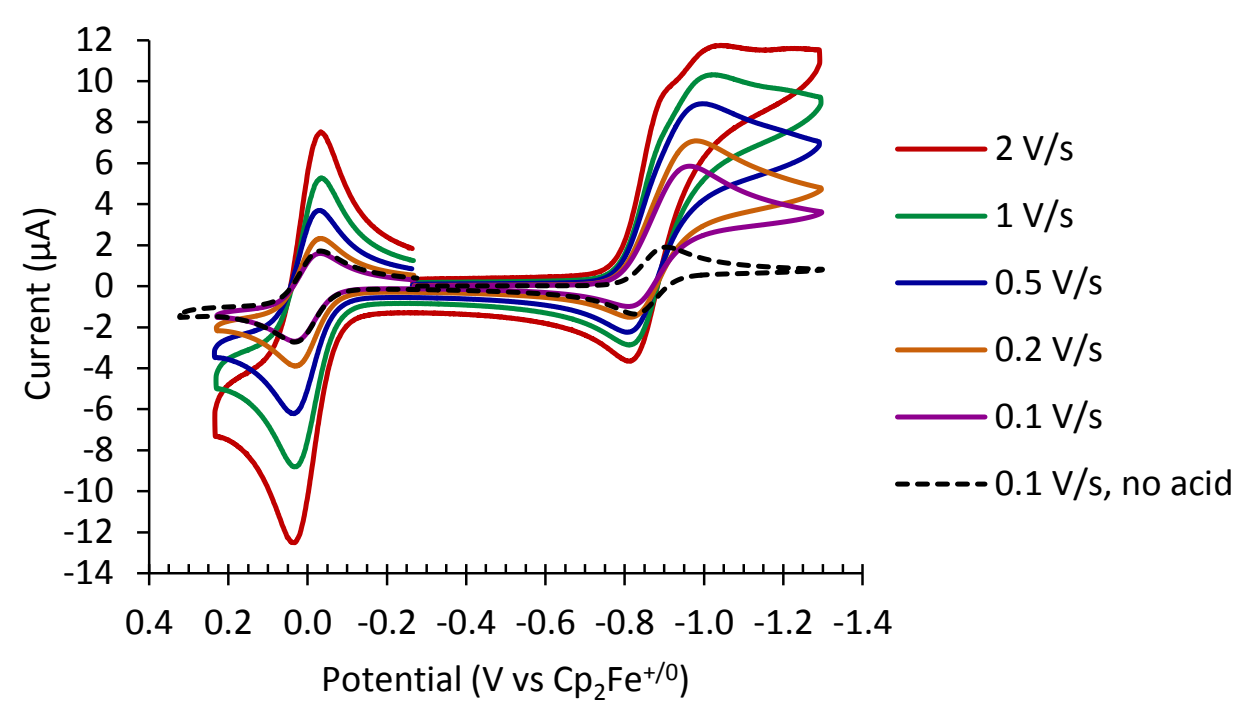

Figure S7. Cyclic voltammograms of $\left[\mathrm{Co}^{\mathrm{II}}\left(\mathrm{P}^{\mathrm{tBu}}{ }_{2} \mathrm{~N}^{\mathrm{Ph}}{ }_{2}\right)\left(\mathrm{CH}_{3} \mathrm{CN}\right)_{3}\right]^{2+}(1 \quad \mathrm{mM})$ and $p$-bromoanilinium tetrafluoroborate $(3 \mathrm{mM})$ at varying scan rates. Conditions: $0.2 \mathrm{M} \mathrm{NBu}_{4} \mathrm{BF}_{4}$ acetonitrile solution, $1 \mathrm{~mm}$ dia. glassy carbon working electrode.
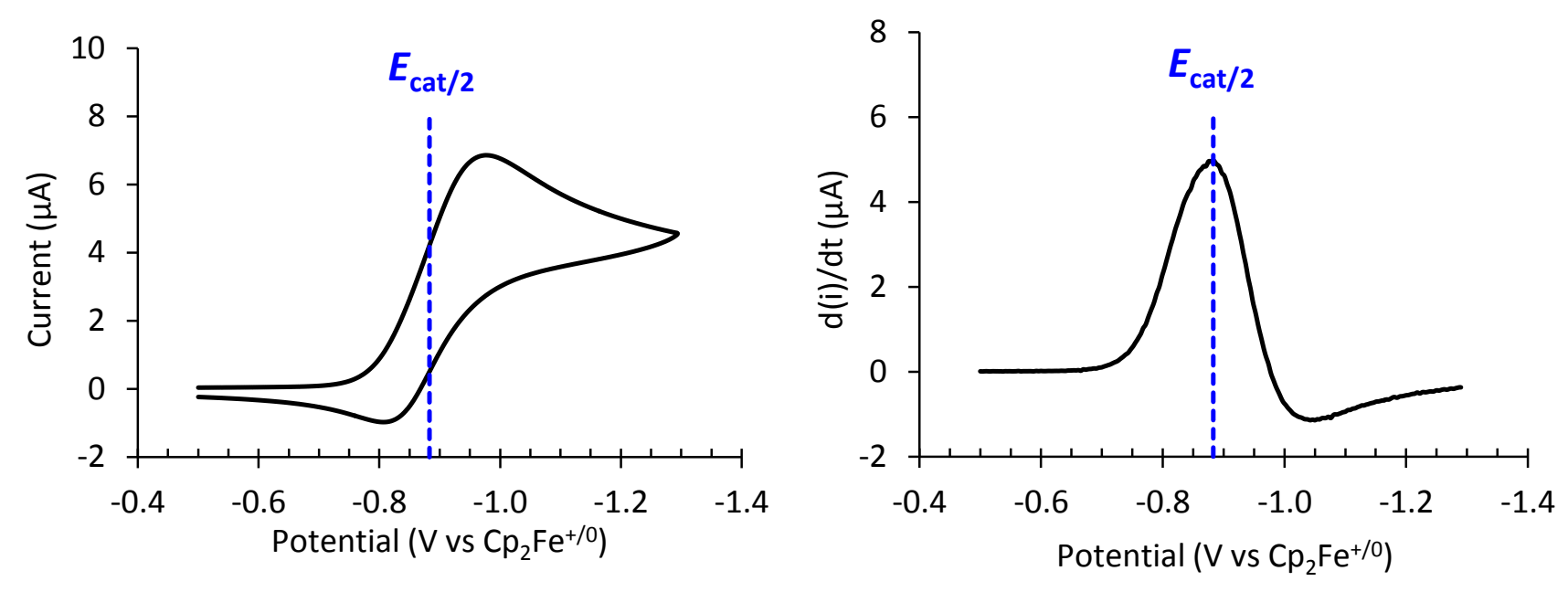

Figure S8. Cyclic voltammogram (left) and first-derivative trace (right) of $\left[\mathrm{Co}^{\mathrm{II}}\left(\mathrm{P}^{t \mathrm{Bu}}{ }_{2} \mathrm{~N}^{\mathrm{Ph}}{ }_{2}\right)\left(\mathrm{CH}_{3} \mathrm{CN}\right)_{3}\right]^{2+}(1 \mathrm{mM})$

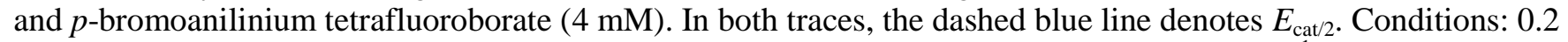
$\mathrm{M} \mathrm{NBu}_{4} \mathrm{BF}_{4}$ acetonitrile solution, $1 \mathrm{~mm}$ diameter glassy carbon working electrode, $v=0.1 \mathrm{~V} \mathrm{~s}^{-1}$. 


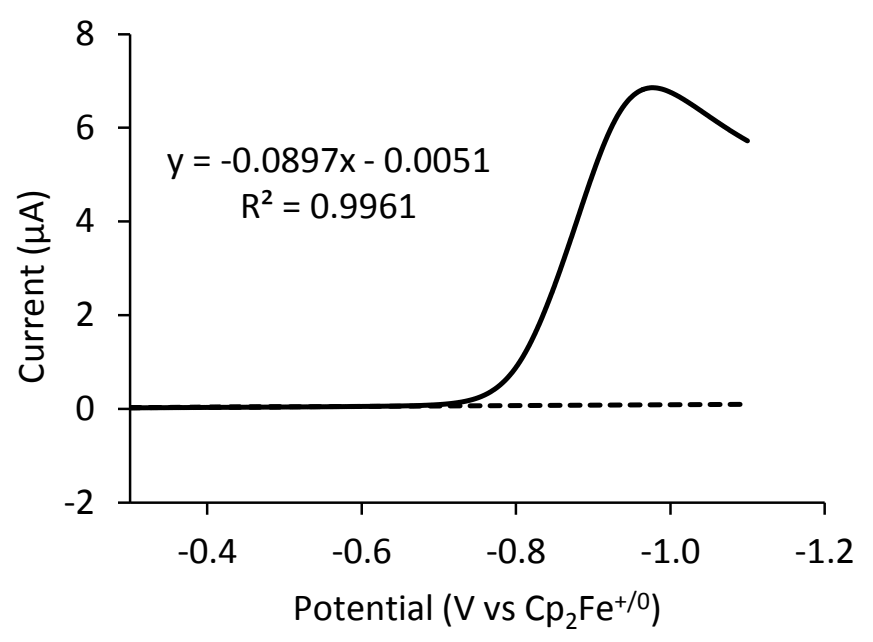

Figure S9. Cyclic voltammogram of $\left[\mathrm{Co}^{\mathrm{II}}\left(\mathrm{P}^{t \mathrm{Bu}}{ }_{2} \mathrm{~N}^{\mathrm{Ph}}{ }_{2}\right)\left(\mathrm{CH}_{3} \mathrm{CN}\right)_{3}\right]^{2+}(1 \mathrm{mM})$ and $p$-bromoanilinium tetrafluoroborate $(4 \mathrm{mM})$ showing the linear extrapolation of the background capacitive current used for backgroudn subtraction in FOWA. Conditions: $0.2 \mathrm{M} \mathrm{NBu}_{4} \mathrm{BF}_{4}$ acetonitrile solution, $1 \mathrm{~mm}$ diameter glassy carbon working electrode, $v=$ $0.1 \mathrm{~V} \mathrm{~s}^{-1}$.

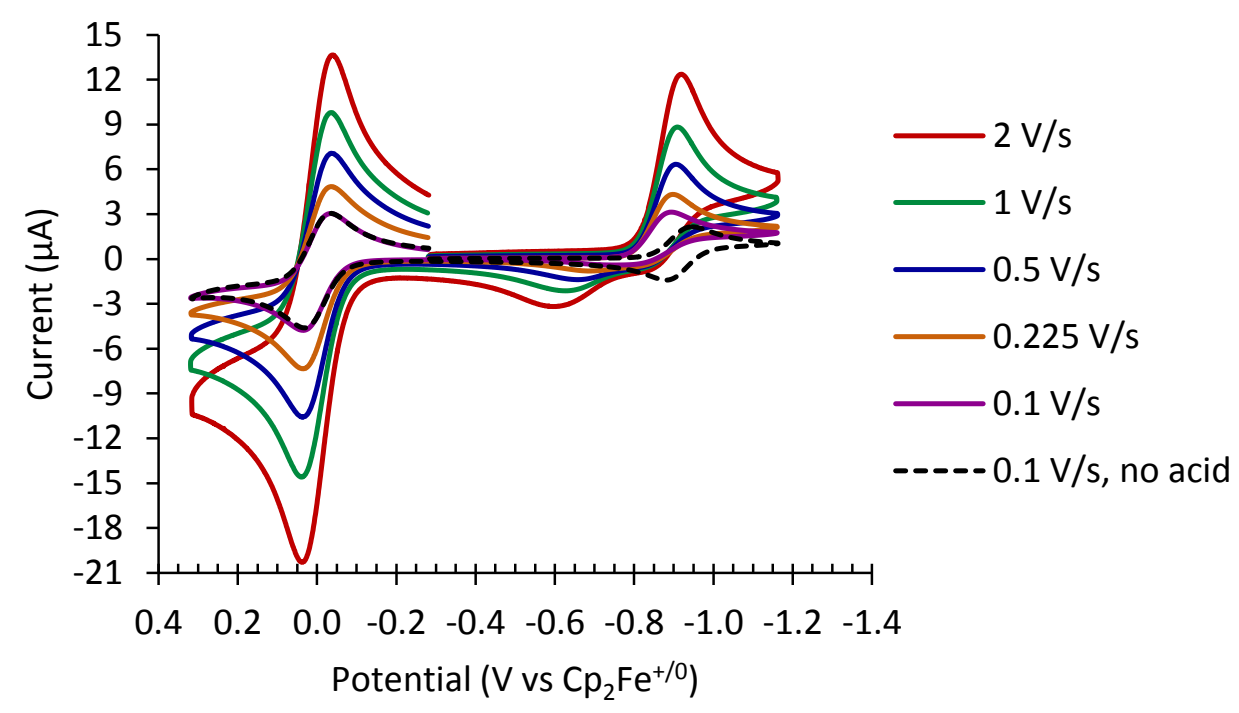

Figure S10. Cyclic voltammograms of $\mathrm{Co}^{\mathrm{II}}\left(\mathrm{dmgBF}_{2}\right)_{2}\left(\mathrm{CH}_{3} \mathrm{CN}\right)_{2}(1 \mathrm{mM})$ and anilinium tetrafluoroborate $(1 \mathrm{mM})$ at varying scan rates. Conditions: $0.2 \mathrm{M} \mathrm{NBu}_{4} \mathrm{BF}_{4}$ acetonitrile solution, $1 \mathrm{~mm}$ dia. glassy carbon working electrode. 


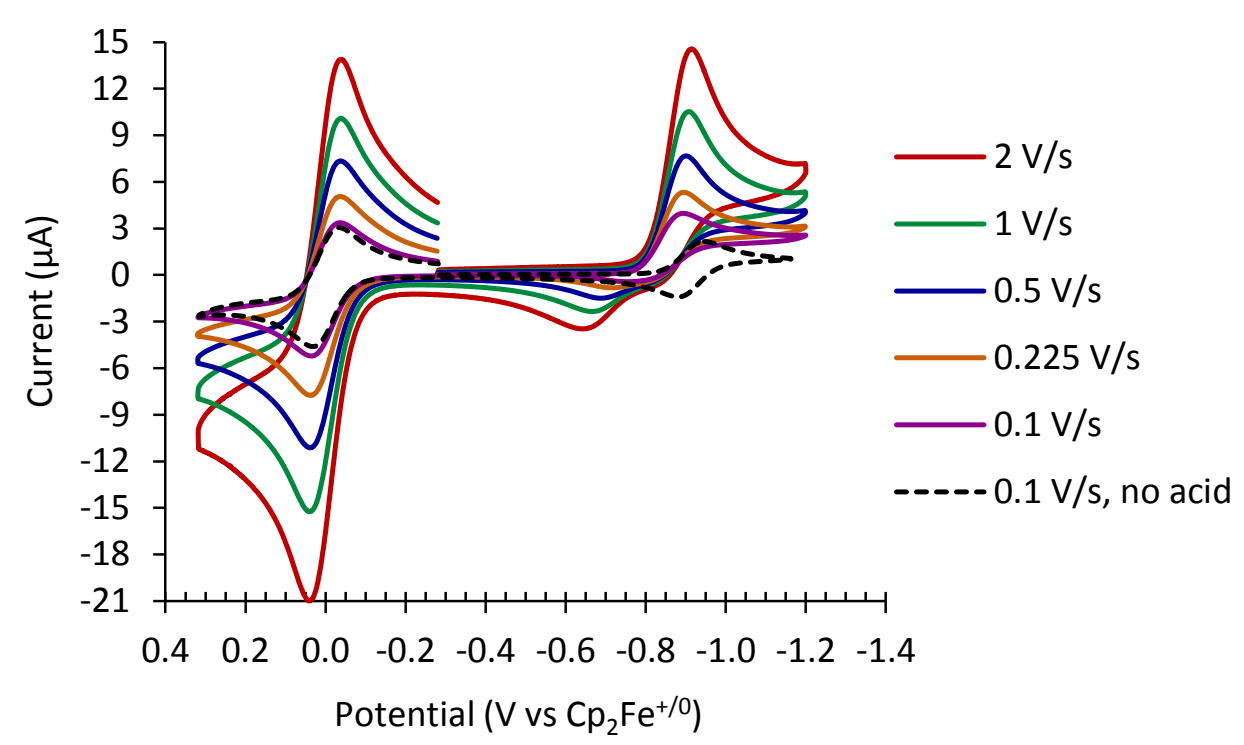

Figure S11. Cyclic voltammograms of $\mathrm{Co}^{\mathrm{II}}\left(\mathrm{dmgBF}_{2}\right)_{2}\left(\mathrm{CH}_{3} \mathrm{CN}\right)_{2}(1 \mathrm{mM})$ and anilinium tetrafluoroborate $(2 \mathrm{mM})$ at varying scan rates. Conditions: $0.2 \mathrm{M} \mathrm{NBu}_{4} \mathrm{BF}_{4}$ acetonitrile solution, $1 \mathrm{~mm}$ dia. glassy carbon working electrode.

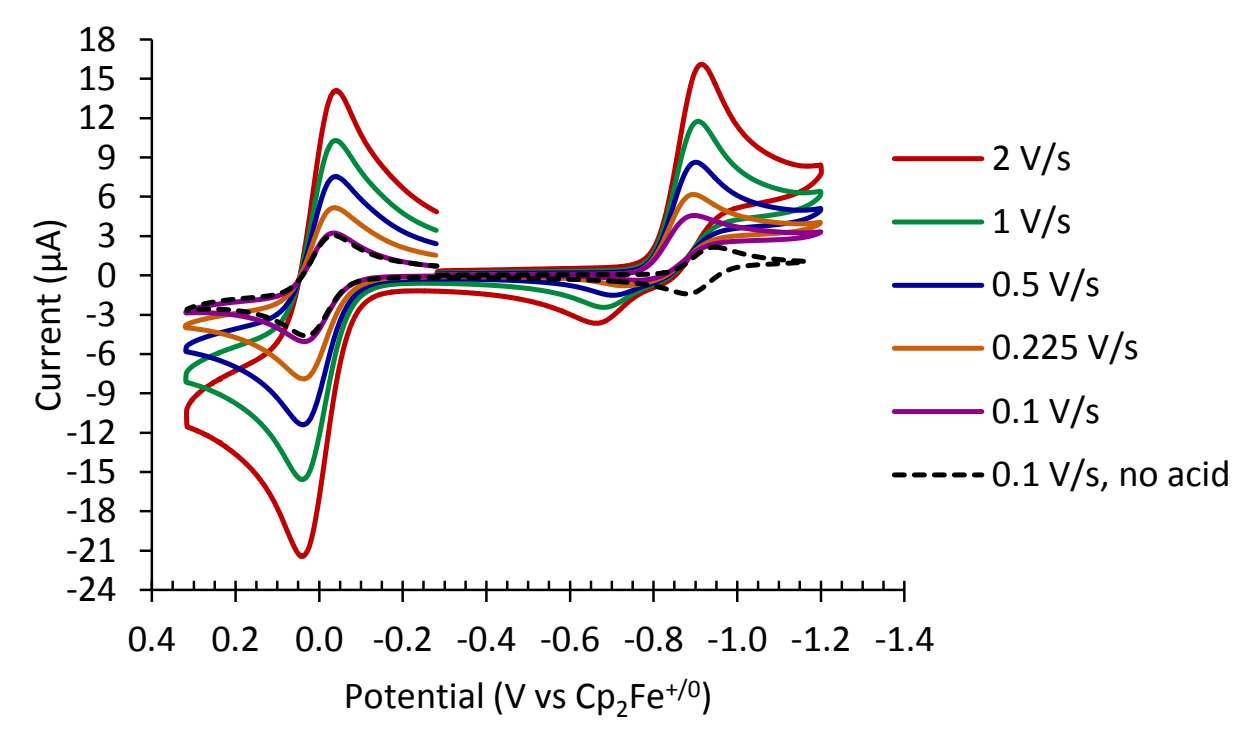

Figure S12. Cyclic voltammograms of $\mathrm{Co}^{\mathrm{II}}\left(\mathrm{dmgBF}_{2}\right)_{2}\left(\mathrm{CH}_{3} \mathrm{CN}\right)_{2}(1 \mathrm{mM})$ and anilinium tetrafluoroborate $(3 \mathrm{mM})$ at varying scan rates. Conditions: $0.2 \mathrm{M} \mathrm{NBu}_{4} \mathrm{BF}_{4}$ acetonitrile solution, $1 \mathrm{~mm}$ dia. glassy carbon working electrode. 


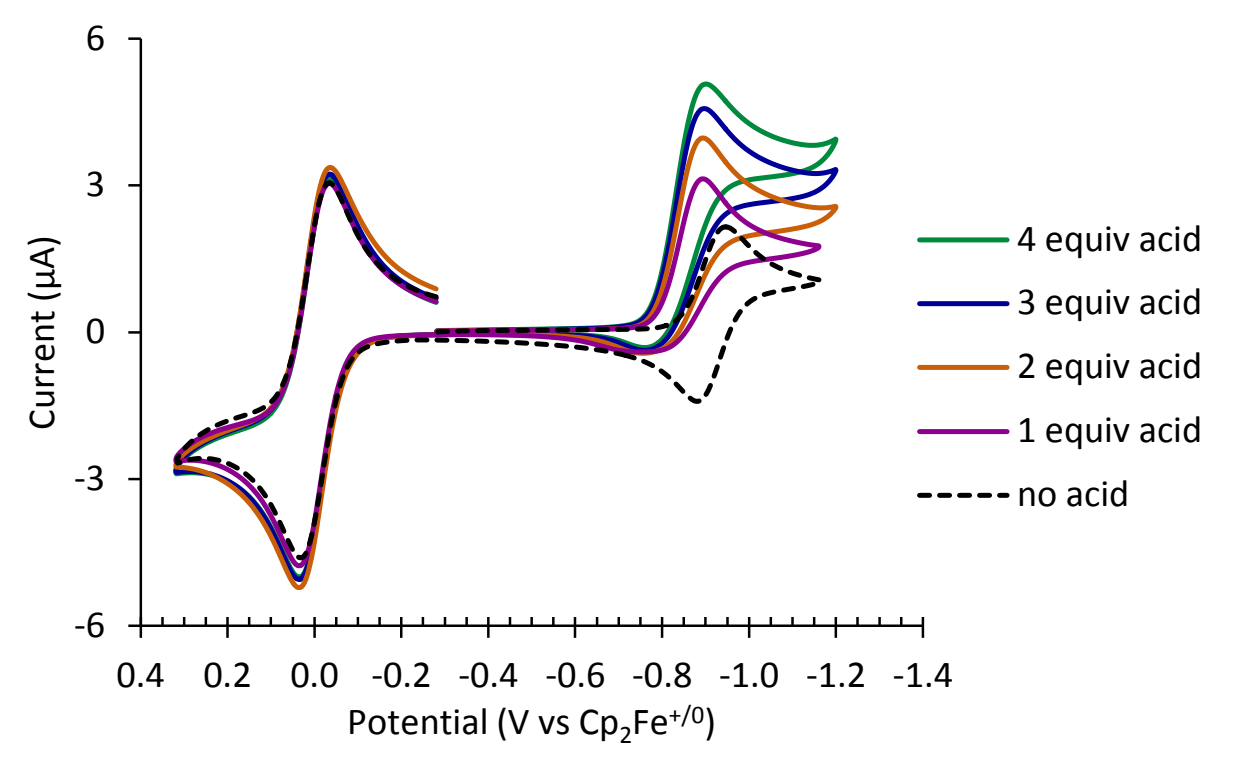

Figure S13. Cyclic voltammograms of $\mathrm{Co}^{\mathrm{II}}\left(\mathrm{dmgBF}_{2}\right)_{2}\left(\mathrm{CH}_{3} \mathrm{CN}\right)_{2}(1 \mathrm{mM})$ and varying concentrations of anilinium tetrafluoroborate $(1-4 \mathrm{mM})$ at $v=0.1 \mathrm{~V} \mathrm{~s}^{-1}$. Conditions: $0.2 \mathrm{M} \mathrm{NBu}_{4} \mathrm{BF}_{4}$ acetonitrile solution, $1 \mathrm{~mm}$ dia. glassy carbon working electrode.
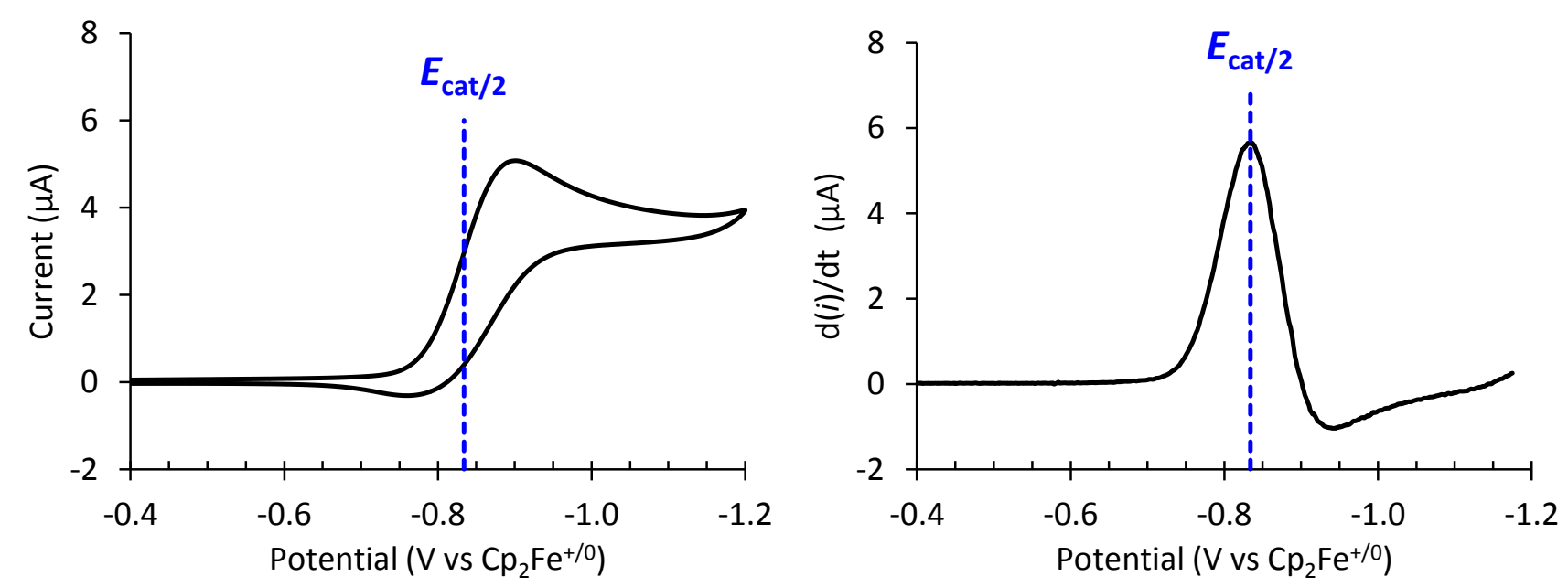

Figure S14. Cyclic voltammogram (left) and first-derivative trace (right) of $\mathrm{Co}^{\mathrm{II}}\left(\mathrm{dmgBF}_{2}\right)_{2}\left(\mathrm{CH}_{3} \mathrm{CN}\right)_{2}(1 \mathrm{mM})$ and anilinium tetrafluoroborate $(4 \mathrm{mM})$. In both traces, the dashed blue line denotes $E_{\text {cat } / 2}$. Conditions: $0.2 \mathrm{M} \mathrm{NBu}_{4} \mathrm{BF}_{4}$ acetonitrile solution, $1 \mathrm{~mm}$ diameter glassy carbon working electrode, $v=0.1 \mathrm{~V} \mathrm{~s}^{-1}$. 


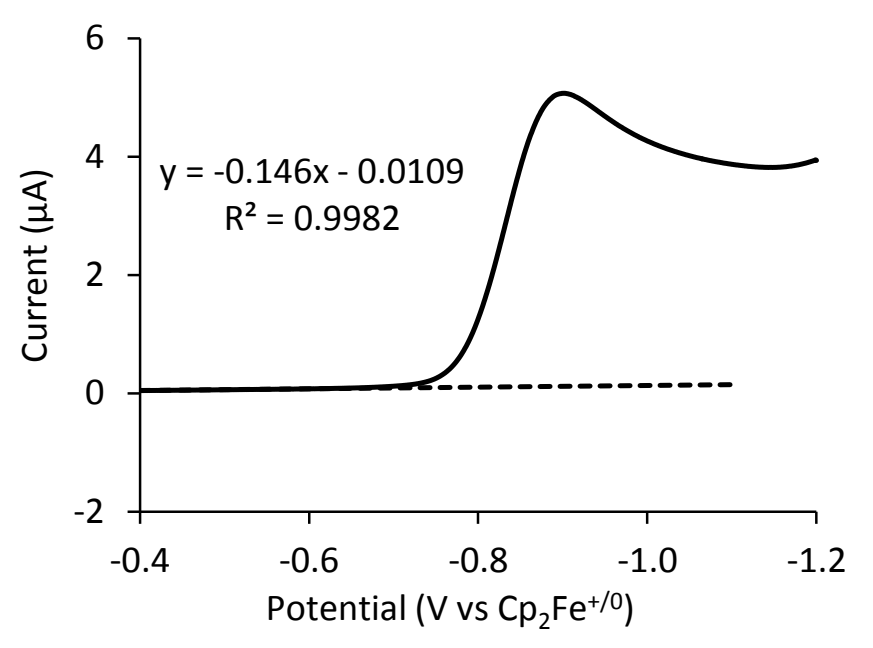

Figure S15. Cyclic voltammogram of $\mathrm{Co}^{\mathrm{II}}\left(\mathrm{dmgBF}_{2}\right)_{2}\left(\mathrm{CH}_{3} \mathrm{CN}\right)_{2}(1 \mathrm{mM})$ and anilinium tetrafluoroborate $(4 \mathrm{mM})$ showing the linear extrapolation of the background capacitive current used for background subtraction in FOWA. Conditions: $0.2 \mathrm{M} \mathrm{NBu}_{4} \mathrm{BF}_{4}$ acetonitrile solution, $1 \mathrm{~mm}$ diameter glassy carbon working electrode, $v=0.1 \mathrm{~V} \mathrm{~s}^{-1}$.

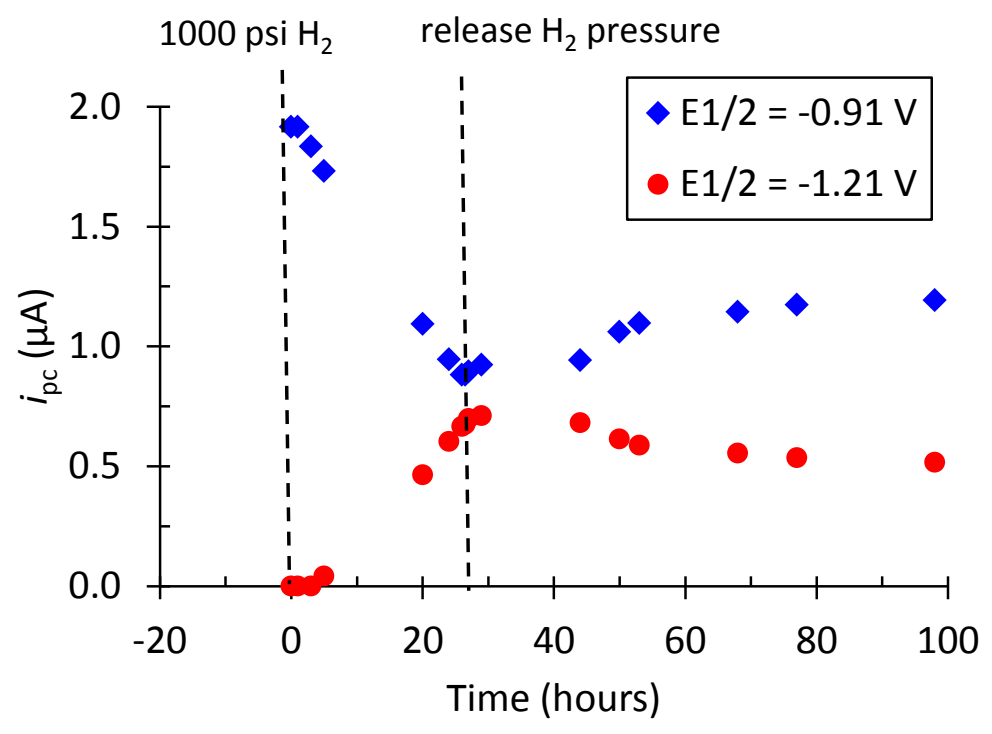

Figure S16. Plot of $i_{\mathrm{pc}}$ versus time for $\mathrm{Co}^{\mathrm{II}}\left(\mathrm{dmgBF}_{2}\right)_{2}\left(\mathrm{CH}_{3} \mathrm{CN}\right)_{2}$ upon pressurizing with 1000 psi $\mathrm{H}_{2}$ and upon releasing the $\mathrm{H}_{2}$ pressure. Conditions: $1 \mathrm{mM}[\mathrm{Co}], 0.2 \mathrm{M} \mathrm{NBu}_{4} \mathrm{PF}_{6}$ acetonitrile solution, $1 \mathrm{~mm}$ diameter glassy carbon working electrode, $v=0.1 \mathrm{~V} \mathrm{~s}^{-1}$. 
Table S2. Energies from DFT calculations.

\begin{tabular}{|c|c|c|c|}
\hline Compound & $\begin{array}{c}G_{\text {corr }} \\
\left(\text { hartree molecule }{ }^{-1}\right)^{a}\end{array}$ & $\begin{array}{c}E_{\mathrm{MeCN}} \\
\left(\text { hartree molecule }{ }^{-1}\right)^{b}\end{array}$ & $\begin{array}{c}G_{\mathrm{MeCN}} \\
\left(\mathrm{kcal} \mathrm{mol}^{-1}\right)^{c}\end{array}$ \\
\hline$\left[\mathrm{Co}^{\mathrm{II}}\left(\mathrm{P}^{\mathrm{tBu}}{ }_{2} \mathrm{~N}_{2}{ }_{2}\right)\left(\mathrm{CH}_{3} \mathrm{CN}\right)_{3}\right]^{2+}$ & 0.621288 & -2277.91896867 & -1429025.86 \\
\hline$\left[\mathrm{HCo}^{\mathrm{III}}\left(\mathrm{P}^{\mathrm{Bu}}{ }_{2} \mathrm{~N}^{\mathrm{Ph}}\right)\left(\mathrm{CH}_{3} \mathrm{CN}\right)_{3}\right]^{2+}$ & 0.631994 & -2278.51208600 & -1429391.33 \\
\hline$\left[\mathrm{HCo}{ }^{\mathrm{II}}\left(\mathrm{P}^{\mathrm{tBu}}{ }_{2} \mathrm{~N}^{\mathrm{Ph}}{ }_{2}\right)\left(\mathrm{CH}_{3} \mathrm{CN}\right)_{2}\right]^{+}$ & 0.585263 & -2145.52431563 & -1345969.57 \\
\hline $\mathrm{Co}^{\mathrm{II}}\left(\mathrm{dmgBF}_{2}\right)\left(\mathrm{CH}_{3} \mathrm{CN}\right)$ & 0.228869 & -1563.68776987 & -981085.26 \\
\hline $\mathrm{HCo}^{\mathrm{IIII}}\left(\mathrm{dmgBF}_{2}\right)\left(\mathrm{CH}_{3} \mathrm{CN}\right)$ & 0.239857 & -1564.28180544 & -981451.13 \\
\hline$\left[\mathrm{Co} \text { II }(\text { dppe })_{2}\left(\mathrm{CH}_{3} \mathrm{CN}\right)\right]^{2+d}$ & 0.811359 & -3662.55835302 & -2297780.91 \\
\hline$\left[\mathrm{HCo}(\mathrm{dppe})_{2}\left(\mathrm{CH}_{3} \mathrm{CN}\right)\right]^{2+d}$ & 0.824786 & -3663.16516189 & -2298153.26 \\
\hline$\left[\mathrm{HCo}^{\mathrm{II}}(\mathrm{dppe})_{2}\right]^{+d}$ & 0.772577 & -3530.17092014 & -2214730.88 \\
\hline
\end{tabular}

${ }^{a}$ Thermal correction to the Gibbs Free Energy (gas phase). ${ }^{b}$ Electronic energy in acetonitrile solution. ${ }^{c}$ Gibbs Free Energy (MeCN solution). $G_{\mathrm{MeCN}}=\left(G_{\mathrm{corr}}+E_{\mathrm{MeCN}}\right) \times 627.509469 \mathrm{kcal} \mathrm{mol}^{-1} .{ }^{d}$ The free energy was previously calculated (Inorg. Chem. 2013, 52, 14391-14403).

\section{Thermochemical Cycles}

In order to complete the thermochemcal cycles tabulated below, constants for the following half reactions associated with cleavage of $\mathrm{H}_{2}$ in acetonitrile solution are required:

$\begin{array}{ll}\mathrm{H}_{2} \rightarrow \mathrm{H}^{+}+\mathrm{H}^{-} & \Delta G^{\circ}=76.0 \mathrm{kcal} \mathrm{mol}^{-1} \\ \mathrm{H}_{2} \rightarrow 2 \mathrm{H}^{\circ} & \Delta G^{\circ}=103.6 \mathrm{kcal} \mathrm{mol}^{-1} \\ \mathrm{H}^{+}+\mathrm{e}^{-} \rightarrow \mathrm{H}^{\circ} & \Delta G^{\circ}=53.6 \mathrm{kcal} \mathrm{mol}^{-1} \\ \mathrm{H}^{\circ}+\mathrm{e}^{-} \rightarrow \mathrm{H}^{-} & \Delta G^{\circ}=26.0 \mathrm{kcal} \mathrm{mol}^{-1}\end{array}$

These constants are determined as free energies relative to the $\mathrm{Cp}_{2} \mathrm{Fe}^{+/ 0}$ couple. ${ }^{1-2}$ Errors in the absolute values of these constants are not considered here, but will not change the relative thermochemical values derived from them.

1. Wayner, D. D. M.; Parker, V. D. Acc. Chem. Res. 1993, 26, 287-294.

2. Ellis, W. W.; Raebiger, J. W.; Curtis, C. J.; Bruno, J. W.; DuBois, D. L. J. Am. Chem. Soc. 2004, 126, 27382743. 


\section{Cycles for Derivatives of $\left[\mathrm{Co}^{\mathrm{II}}\left(\mathrm{P}^{t \mathrm{Bu}}{ }_{2} \mathrm{~N}^{\mathrm{Ph}}\right)\left(\mathrm{CH}_{3} \mathrm{CN}\right)_{3}\right]^{2+}$}

\begin{tabular}{|c|c|c|}
\hline $\begin{aligned} {\left[\mathrm{HCo}^{\mathrm{II}}\left(\mathrm{P}_{2}^{t \mathrm{Bu}}{ }_{2} \mathrm{~N}_{2}^{\mathrm{Ph}}\right)\left(\mathrm{CH}_{3} \mathrm{CN}\right)_{2}\right.} \\
\rightarrow\left[\mathrm{Co}^{\mathrm{II}}\left(\mathrm{P}^{t \mathrm{Bu}}{ }_{2}\right.\right.\end{aligned}$ & $\begin{array}{l}\left.\mathrm{O}^{\mathrm{II}}(\mathrm{dppe})_{2}\left(\mathrm{CH}_{3} \mathrm{CN}\right)\right]^{2+} \\
\left.\left.\mathrm{CH}_{3} \mathrm{CN}\right)_{3}\right]^{2+}+\left[\mathrm{HCo}^{\mathrm{II}}(\mathrm{dppe})_{2}\right]^{+}\end{array}$ & $\Delta G^{\circ}(\mathrm{DFT})=-6.3 \mathrm{kcal} \mathrm{mol}^{-1}$ \\
\hline$\left[\mathrm{HCo}^{\mathrm{II}}(\mathrm{dppe})_{2}\right]^{+}+\mathrm{CH}_{3} \mathrm{CN}$ & $\left.(\mathrm{dppe})_{2}\left(\mathrm{CH}_{3} \mathrm{CN}\right)\right]^{+}+\mathrm{H}^{-}$ & $\Delta G_{\mathrm{H}-}^{\circ}=60.5 \mathrm{kcal} \mathrm{mol}^{-1}$ \\
\hline$\left[\mathrm{HCo}{ }^{\mathrm{II}}\left(\mathrm{P}_{2}^{t \mathrm{Bu}}{ }_{2} \mathrm{~N}_{2}{ }_{2}\right)\left(\mathrm{CH}_{3} \mathrm{CN}\right)_{2}\right.$ & $\mathrm{H}_{3} \mathrm{CN} \rightarrow\left[\mathrm{Co}^{\mathrm{II}}\left(\mathrm{P}_{2}^{t \mathrm{Bu}} \mathrm{N}_{2} \mathrm{Ph}_{2}\right)\left(\mathrm{CH}_{3} \mathrm{CN}\right)_{3}\right]^{2+}+\mathrm{H}^{-}$ & $\Delta G_{\mathrm{H}-}^{\circ}=54.2 \mathrm{kcal} \mathrm{mol}^{-1}$ \\
\hline $\begin{array}{r}{\left[\mathrm{HCo}^{\mathrm{IIII}}\left(\mathrm{P}_{2}^{t \mathrm{Bu}}{ }_{2} \mathrm{~N}_{2}^{\mathrm{Ph}}\right)\left(\mathrm{CH}_{3} \mathrm{CN}\right)\right.} \\
\rightarrow\left[\mathrm{HCo}^{\mathrm{II}}\left(\mathrm{P}^{t \mathrm{Bu}}\right.\right.\end{array}$ & $\begin{array}{l}\left.\mathrm{HCo}^{\mathrm{II}}(\mathrm{dppe})_{2}\right]^{+} \\
\left.\left.\mathrm{CH}_{3} \mathrm{CN}\right)_{2}\right]^{+}+\left[\mathrm{HCo}^{\mathrm{III}}(\mathrm{dppe})_{2}\left(\mathrm{CH}_{3} \mathrm{CN}\right)\right]^{2+}\end{array}$ & $\Delta G^{\circ}(\mathrm{DFT})=-0.6 \mathrm{kcal} \mathrm{mol}^{-1}$ \\
\hline$\left[\mathrm{HCo}^{\mathrm{III}}(\mathrm{dppe})_{2}\left(\mathrm{CH}_{3} \mathrm{CN}\right)\right]^{2+}$ & {$\left[\mathrm{HCo}^{\mathrm{II}}(\mathrm{dppe})_{2}\right]^{+}+\mathrm{CH}_{3} \mathrm{CN}$} & $\Delta G^{\circ}=-23.06 E^{\circ}=19.1 \mathrm{kcal} \mathrm{mol}^{-1}$ \\
\hline$\left[\mathrm{HCo}^{\mathrm{III}}\left(\mathrm{P}_{2}^{\mathrm{tBu}}{ }_{2} \mathrm{~N}_{2}{ }^{\mathrm{Ph}}\right)\left(\mathrm{CH}_{3} \mathrm{CN}\right)\right.$ & $\rightarrow\left[\mathrm{HCo}^{\mathrm{II}}\left(\mathrm{P}^{t \mathrm{Bu}}{ }_{2} \mathrm{~N}_{2}^{\mathrm{Ph}}\right)\left(\mathrm{CH}_{3} \mathrm{CN}\right)_{2}\right]^{+}+\mathrm{CH}_{3} \mathrm{CN}$ & $E^{\circ}=\Delta G^{\circ} /(-23.06)=-0.80 \mathrm{~V}$ \\
\hline$\left[\mathrm{HCo}^{\mathrm{III}}\right]^{2+}+\mathrm{e}^{-} \rightarrow\left[\mathrm{HCo}^{\mathrm{II}}\right]^{+}$ & $\Delta G^{\circ}=-23.06 E^{\circ}=18.4 \mathrm{kcal} \mathrm{mol}^{-1}$ & \\
\hline$\left[\mathrm{HCo}^{\mathrm{II}}\right]^{+} \rightarrow\left[\mathrm{Co}^{\mathrm{II}}\right]^{2+}+\mathrm{H}^{-}$ & $\Delta G_{\mathrm{H}-}^{\circ}=54.2 \mathrm{kcal} \mathrm{mol}^{-1}$ & \\
\hline $\mathrm{H}^{-} \rightarrow \mathrm{H}^{\cdot}+\mathrm{e}^{-}$ & $\Delta G^{\circ}=-26.0 \mathrm{kcal} \mathrm{mol}{ }^{-1}$ & \\
\hline$\left[\mathrm{HCo}^{\mathrm{III}}\right]^{2+} \rightarrow\left[\mathrm{Co}^{\mathrm{II}}\right]^{2+}+\mathrm{H}^{\cdot}$ & $\Delta G_{\mathrm{H} \cdot}^{\circ}=46.6 \mathrm{kcal} \mathrm{mol}^{-1}$ & \\
\hline$\left[\mathrm{HCo}^{\mathrm{III}}\right]^{2+} \rightarrow\left[\mathrm{Co}^{\mathrm{II}}\right]^{2+}+\mathrm{H}^{\cdot}$ & $\Delta G_{\mathrm{H} \cdot}^{\circ}=46.6 \mathrm{kcal} \mathrm{mol}^{-1}$ & \\
\hline$\left[\mathrm{Co}^{\mathrm{II}}\right]^{2+}+\mathrm{e}^{-} \rightarrow\left[\mathrm{Co}^{\mathrm{I}}\right]^{+}$ & $\Delta G^{\circ}=-23.06 E^{\circ}=20.1 \mathrm{kcal} \mathrm{mol}^{-1}$ & \\
\hline $\mathrm{H}^{\cdot} \rightarrow \mathrm{H}^{+}+\mathrm{e}^{-}$ & $\Delta G^{\circ}=-53.6 \mathrm{kcal} \mathrm{mol}^{-1}$ & \\
\hline$\left[\mathrm{HCo}^{\mathrm{III}}\right]^{2+} \rightarrow\left[\mathrm{Co}^{\mathrm{I}}\right]^{+}+\mathrm{H}^{+}$ & $\mathrm{p} K_{\mathrm{a}}=\Delta G^{\circ} / 1.364=9.6$ & \\
\hline$\left[\mathrm{HCo}^{\mathrm{III}}\right]^{2+} \rightarrow\left[\mathrm{Co}^{\mathrm{I}}\right]^{+}+\mathrm{H}^{+}$ & $\Delta G^{\circ}=1.364 \mathrm{p} K_{\mathrm{a}}=13.1 \mathrm{kcal} \mathrm{mol}^{-1}$ & \\
\hline$\left[\mathrm{HCo}^{\mathrm{II}}\right]^{+} \rightarrow\left[\mathrm{HCo}^{\mathrm{III}}\right]^{2+}+\mathrm{e}^{-}$ & $\Delta G^{\circ}=+23.06 E^{\circ}=-18.4 \mathrm{kcal} \mathrm{mol}^{-1}$ & \\
\hline $\mathrm{H}^{+}+\mathrm{e}^{-} \rightarrow \mathrm{H}^{\cdot}$ & $\Delta G^{\circ}=53.6 \mathrm{kcal} \mathrm{mol}^{-1}$ & \\
\hline$\left[\mathrm{HCo}^{\mathrm{II}}\right]^{+} \rightarrow\left[\mathrm{Co}^{\mathrm{I}}\right]^{+}+\mathrm{H}^{\cdot}$ & $\Delta G_{\mathrm{H} \cdot}^{\circ}=48.3 \mathrm{kcal} \mathrm{mol}^{-1}$ & \\
\hline$\left[\mathrm{HCo}^{\mathrm{III}}\right]^{2+} \rightarrow\left[\mathrm{Co}^{\mathrm{II}}\right]^{2+}+\mathrm{H}^{\cdot}$ & $\Delta G_{\mathrm{H}_{\bullet}}^{\circ}=46.6 \mathrm{kcal} \mathrm{mol}^{-1}$ & \\
\hline$\left[\mathrm{Co}^{\mathrm{II}}\right]^{2+} \rightarrow\left[\mathrm{Co}^{\mathrm{III}}\right]^{3+}+\mathrm{e}^{-}$ & $\Delta G^{\circ}=+23.06 E^{\circ}=7.8 \mathrm{kcal} \mathrm{mol}^{-1}$ & \\
\hline $\mathrm{H}^{\cdot}+\mathrm{e}^{-} \rightarrow \mathrm{H}^{-}$ & $\Delta G^{\circ}=26.0 \mathrm{kcal} \mathrm{mol}^{-1}$ & \\
\hline$\left[\mathrm{HCo}^{\mathrm{III}}\right]^{2+} \rightarrow\left[\mathrm{Co}^{\mathrm{III}}\right]^{3+}+\mathrm{H}^{-}$ & $\Delta G_{\mathrm{H}-}^{\circ}=80.4 \mathrm{kcal} \mathrm{mol}^{-1}$ & \\
\hline
\end{tabular}




\section{Cycles for Derivatives of $\mathrm{Co}^{\mathrm{II}}\left(\mathrm{dmgBF}_{2}\right)_{2}\left(\mathrm{CH}_{3} \mathrm{CN}\right)_{2}$}

\begin{tabular}{|c|c|c|}
\hline \multicolumn{2}{|c|}{$\rightarrow \mathrm{Co}^{\mathrm{II}}\left(\mathrm{dmgBF}_{2}\right)\left(\mathrm{CH}_{3} \mathrm{CN}\right)+\left[\mathrm{HCo}^{\mathrm{III}}(\mathrm{dppe})_{2}\left(\mathrm{CH}_{3} \mathrm{CN}\right)\right]^{2+}$} & $\Delta G^{\circ}(\mathrm{DFT})=-6.5 \mathrm{kcal} \mathrm{mol}^{-1}$ \\
\hline \multicolumn{2}{|c|}{$\left[\mathrm{HCo}^{\mathrm{III}}(\mathrm{dppe})_{2}\left(\mathrm{CH}_{3} \mathrm{CN}\right)\right]^{2+} \rightarrow\left[\mathrm{Co}^{\mathrm{II}}(\mathrm{dppe})_{2}\left(\mathrm{CH}_{3} \mathrm{CN}\right)\right]^{2+}+\mathrm{H}^{\cdot}$} & $\Delta G_{\mathrm{H}^{\circ}}^{\circ}=53.7 \mathrm{kcal} \mathrm{mol}^{-1}$ \\
\hline \multicolumn{2}{|c|}{$\mathrm{HCo}^{\mathrm{III}}\left(\mathrm{dmgBF}_{2}\right)_{2}\left(\mathrm{CH}_{3} \mathrm{CN}\right) \rightarrow \mathrm{Co}^{\mathrm{II}}\left(\mathrm{dmgBF}_{2}\right)_{2}\left(\mathrm{CH}_{3} \mathrm{CN}\right)+\mathrm{H}^{\cdot}$} & $\Delta G_{\mathrm{H} \cdot}^{\circ}=47.2 \mathrm{kcal} \mathrm{mol}^{-1}$ \\
\hline $\mathrm{HCo}^{\mathrm{III}} \rightarrow \mathrm{Co}^{\mathrm{II}}+\mathrm{H}^{\cdot}$ & $\Delta G_{\mathrm{H} \bullet}^{\circ}=47.2 \mathrm{kcal} \mathrm{mol}^{-1}$ & \\
\hline $\mathrm{Co}^{\mathrm{II}}+\mathrm{e}^{-} \rightarrow\left[\mathrm{Co}^{\mathrm{I}}\right]^{-}$ & $\Delta G^{\circ}=-23.06 E^{\circ}=21.0 \mathrm{kcal} \mathrm{mol}^{-1}$ & \\
\hline $\mathrm{H}^{\cdot} \rightarrow \mathrm{H}^{+}+\mathrm{e}^{-}$ & $\Delta G^{\circ}=-53.6 \mathrm{kcal} \mathrm{mol}^{-1}$ & \\
\hline $\mathrm{HCo}^{\mathrm{III}} \rightarrow\left[\mathrm{Co}^{\mathrm{I}}\right]^{-}+\mathrm{H}^{+}$ & $\mathrm{p} K_{\mathrm{a}}=\Delta G^{\circ} / 1.364=10.7$ & \\
\hline $\mathrm{HCo}^{\mathrm{III}} \rightarrow \mathrm{Co}^{\mathrm{II}}+\mathrm{H}^{\cdot}$ & $\Delta G_{\mathrm{H}^{\bullet}}^{\circ}=47.2 \mathrm{kcal} \mathrm{mol}^{-1}$ & \\
\hline$\left[\mathrm{HCo}^{\mathrm{II}}\right]^{-} \rightarrow \mathrm{HCo}^{\mathrm{III}}+\mathrm{e}^{-}$ & $\Delta G^{\circ}=+23.06 E^{\circ}=-17.3 \mathrm{kcal} \mathrm{mol}^{-1}$ & \\
\hline $\mathrm{H}^{\cdot}+\mathrm{e}^{-} \rightarrow \mathrm{H}^{-}$ & $\Delta G^{\circ}=26.0 \mathrm{kcal} \mathrm{mol}^{-1}$ & \\
\hline$\left[\mathrm{HCo}^{\mathrm{II}}\right]^{-} \rightarrow \mathrm{Co}^{\mathrm{II}}+\mathrm{H}^{-}$ & $\Delta G_{\mathrm{H}_{-}}^{\circ}=55.9 \mathrm{kcal} \mathrm{mol}^{-1}$ & \\
\hline $\mathrm{HCo}^{\mathrm{III}} \rightarrow\left[\mathrm{Co}^{\mathrm{I}}\right]^{-}+\mathrm{H}^{+}$ & $\Delta G^{\circ}=1.364 \mathrm{p} K_{\mathrm{a}}=14.6 \mathrm{kcal} \mathrm{mol}^{-1}$ & \\
\hline$\left[\mathrm{HCo}^{\mathrm{II}}\right]^{-} \rightarrow \mathrm{HCo}^{\mathrm{III}}+\mathrm{e}^{-}$ & $\Delta G^{\circ}=+23.06 E^{\circ}=-17.3 \mathrm{kcal} \mathrm{mol}^{-1}$ & \\
\hline $\mathrm{H}^{+}+\mathrm{e}^{-} \rightarrow \mathrm{H}^{\cdot}$ & $\Delta G^{\circ}=53.6 \mathrm{kcal} \mathrm{mol}^{-1}$ & \\
\hline$\left[\mathrm{HCo}^{\mathrm{II}}\right]^{-} \rightarrow\left[\mathrm{Co}^{\mathrm{I}}\right]^{-}+\mathrm{H}^{\cdot}$ & $\Delta G_{\mathrm{H} \cdot}^{\circ}=50.9 \mathrm{kcal} \mathrm{mol}^{-1}$ & \\
\hline $\mathrm{HCo}^{\mathrm{III}} \rightarrow \mathrm{Co}^{\mathrm{II}}+\mathrm{H}^{\cdot}$ & $\Delta G_{\mathrm{H} \cdot}^{\circ}=47.2 \mathrm{kcal} \mathrm{mol}^{-1}$ & \\
\hline $\mathrm{Co}^{\mathrm{II}} \rightarrow\left[\mathrm{Co}^{\mathrm{III}}\right]^{+}+\mathrm{e}^{-}$ & $\Delta G^{\circ}=+23.06 E^{\circ}=3.2 \mathrm{kcal} \mathrm{mol}^{-1}$ & \\
\hline $\mathrm{H}^{\cdot}+\mathrm{e}^{-} \rightarrow \mathrm{H}^{-}$ & $\Delta G^{\circ}=26.0 \mathrm{kcal} \mathrm{mol}^{-1}$ & \\
\hline$\left[\mathrm{HCo}^{\mathrm{III}}\right]^{2+} \rightarrow\left[\mathrm{Co}^{\mathrm{III}}\right]^{3+}+$ & $\Delta G_{\mathrm{H}-}^{\circ}=76.4 \mathrm{kcal} \mathrm{mol}^{-1}$ & \\
\hline
\end{tabular}




\section{Correlation of Reduction Potentials and Heterolytic/Homolytic Co-H Bond Strengths}

Table S3. $\mathrm{Co}^{\mathrm{II} / \mathrm{I}}$ and $\mathrm{Co}^{\mathrm{III} / \mathrm{II}} \mathrm{H}$ Reduction Potentials in Acetonitrile. ${ }^{a}$

\begin{tabular}{|c|c|c|c|}
\hline Complex & $\begin{array}{l}E^{\circ \prime}\left(\mathrm{Co}^{11 / 1}\right) \\
\mathrm{V}\end{array}$ & $\begin{array}{r}E^{\circ \prime}\left(\mathrm{Co}^{\mathrm{IIIIII}} \mathrm{H}\right) \\
\mathbf{V}\end{array}$ & $\begin{array}{l}E^{\circ \prime}\left(\mathrm{Co}^{I I I / I I} \mathrm{H}\right)-E^{\circ \prime}\left(\mathrm{Co}^{I I / T}\right) \\
\mathrm{V}\end{array}$ \\
\hline $\mathrm{HCo}^{\mathrm{III}}\left(\mathrm{dmgBF}_{2}\right)_{2}\left(\mathrm{CH}_{3} \mathrm{CN}\right)$ & -0.91 & -0.75 & 0.16 \\
\hline$\left[\mathrm{HCo}^{\mathrm{III}}\left(\mathrm{P}^{t \mathrm{Bu}}{ }_{2} \mathrm{~N}^{\mathrm{Ph}}{ }_{2}\right)\left(\mathrm{CH}_{3} \mathrm{CN}\right)_{3}\right]^{2+}$ & -0.87 & -0.80 & 0.07 \\
\hline$\left[\mathrm{HCo}^{\mathrm{III}}(\mathrm{dppe})_{2}\left(\mathrm{CH}_{3} \mathrm{CN}\right)\right]^{2+}$ & -0.70 & -0.83 & -0.13 \\
\hline$\left[\mathrm{HCo}^{\mathrm{III}}\left(6 \mathrm{P}_{4} \mathrm{~N}_{2}\right)\left(\mathrm{CH}_{3} \mathrm{CN}\right)\right]^{2+}$ & -0.82 & -1.27 & -0.45 \\
\hline$\left[\mathrm{HCo}^{\mathrm{III}}\left(\mathrm{Cp}^{\mathrm{C} 5 \mathrm{H} 4 \mathrm{~N}}\right)\left(\mathrm{P}^{t \mathrm{Bu}}{ }_{2} \mathrm{~N}^{\mathrm{Ph}}{ }_{2}\right)\right]^{+}$ & -0.95 & -1.61 & -0.66 \\
\hline$\left[\mathrm{HCo}^{\mathrm{III}}\left(5 \mathrm{P}_{4} \mathrm{~N}_{2}\right)\left(\mathrm{CH}_{3} \mathrm{CN}\right)\right]^{2+}$ & -0.82 & -1.55 & -0.73 \\
\hline
\end{tabular}

${ }^{a}$ Additional data that is not shown in Table 1 in the main text.

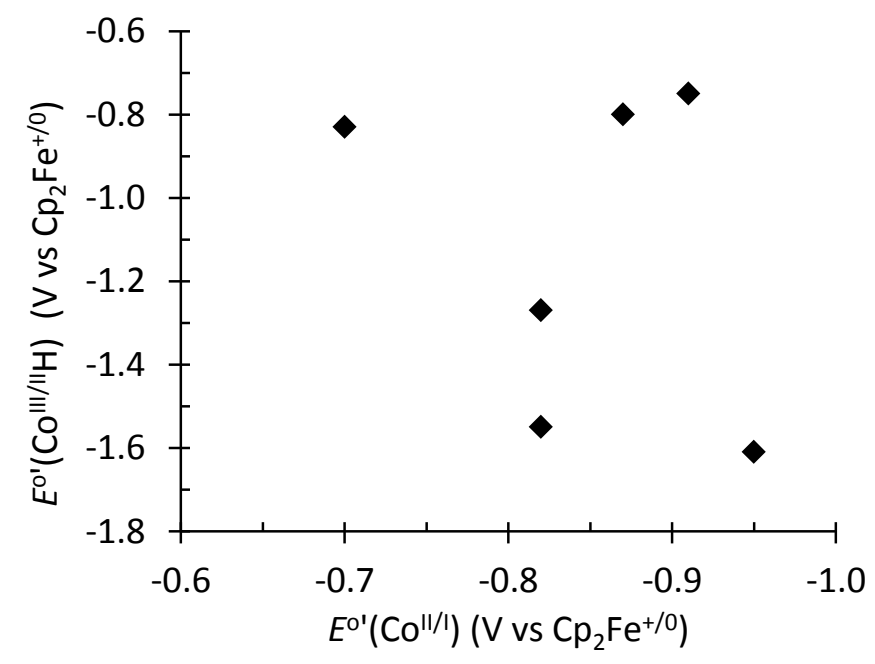

Figure S17. Plot of $E^{\circ \prime}\left(\mathrm{Co}^{\mathrm{III} / \mathrm{II}} \mathrm{H}\right)$ versus $E^{\circ}\left(\mathrm{Co}^{\mathrm{II} / /}\right)$ for the complexes in Tables 1 and $\mathrm{S} 1$. The lack of correlation indictates that the difference between the two couples does not change uniformly as a function of ligand identity. 


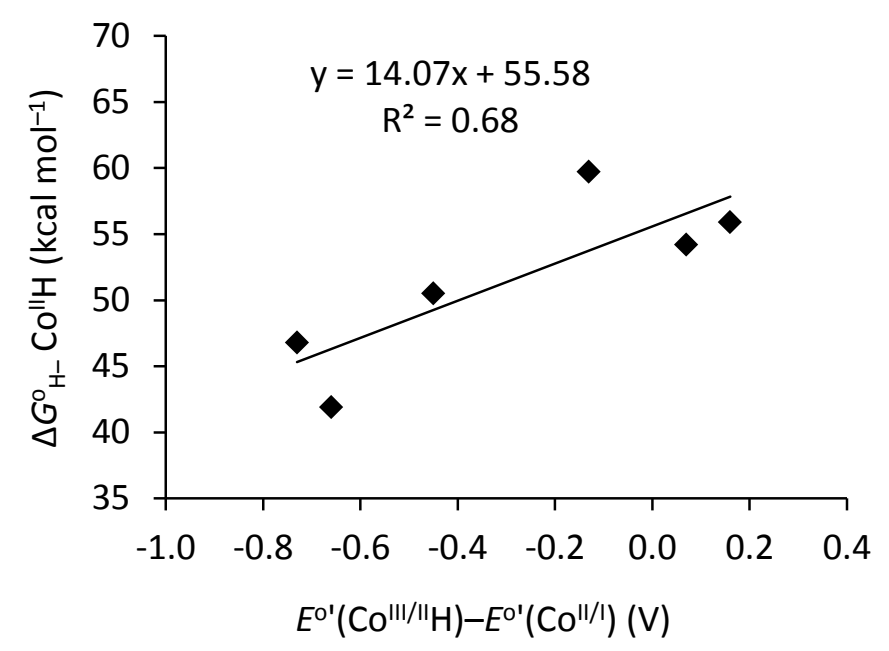

Figure S18. Plot of $\Delta G^{\circ{ }^{\prime}-}$ for $\mathrm{Co}^{\mathrm{II}} \mathrm{H}$ versus $E^{\circ}\left(\mathrm{Co}^{\mathrm{III} / \mathrm{II}} \mathrm{H}\right)-E^{\circ}\left(\mathrm{Co}^{\mathrm{II} / \mathrm{I}}\right)$ for the complexes in Table 1 .

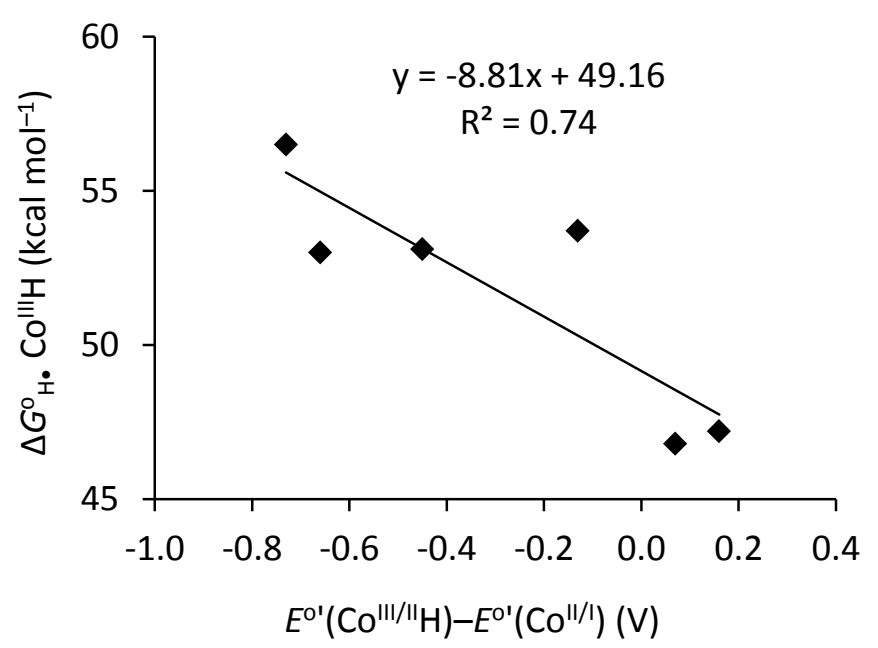

Figure S19. Plot of $\Delta G^{\circ}{ }_{H}$. for $\mathrm{Co}^{\mathrm{III}} \mathrm{H}$ versus $E^{\circ \prime}\left(\mathrm{Co}^{\mathrm{III} / \mathrm{I}} \mathrm{H}\right)-E^{\circ}\left(\mathrm{Co}^{\mathrm{II} / \mathrm{I}}\right)$ for the complexes in Table 1 . 


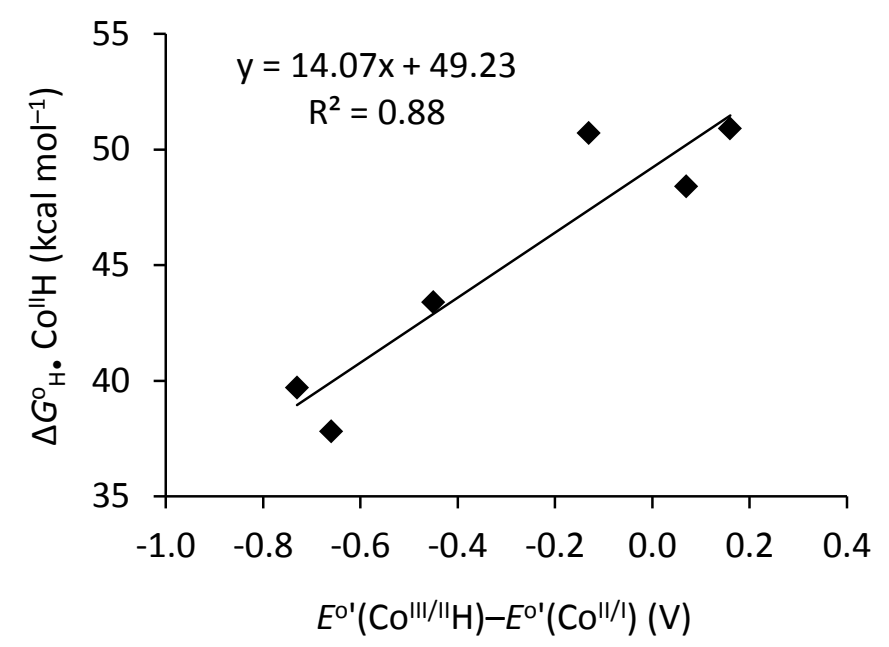

Figure S20. Plot of $\Delta{G^{\circ}}^{\circ}$. for $\mathrm{Co}^{\mathrm{II}} \mathrm{H}$ versus $E^{\circ \prime}\left(\mathrm{Co}^{\mathrm{III/II}} \mathrm{H}\right)-E^{\circ}\left(\mathrm{Co}^{\mathrm{III} / \mathrm{I}}\right)$ for the complexes in Table 1.

\section{XYZ Coordinates for Geometry Optimized Structures.}

$\begin{array}{lrrr}{\left[\mathrm{Co}^{\mathrm{II}}\left(\mathrm{P}^{\mathrm{tBu}}{ }_{2} \mathrm{~N}^{\mathrm{Ph}}\right)\left(\mathrm{CH}_{3} \mathrm{CN}\right)_{3}\right]^{2+}} & \\ \mathrm{Co} & -1.10379 & 0.20012 & 0.67396 \\ \mathrm{~N} & 2.64807 & -0.12555 & -0.08088 \\ \mathrm{~N} & -0.11523 & -0.38047 & -2.47705 \\ \mathrm{~N} & -0.28912 & 0.35800 & 2.64880 \\ \mathrm{~N} & -2.55842 & -0.96189 & 1.25101 \\ \mathrm{~N} & -2.32011 & 1.67105 & 1.07920 \\ \mathrm{P} & 0.42078 & 1.49350 & -0.43105 \\ \mathrm{P} & 0.28926 & -1.51190 & 0.02887 \\ \mathrm{C} & 0.32409 & 3.38704 & -0.49387 \\ \mathrm{C} & 0.39536 & 3.92080 & 0.94263 \\ \mathrm{H} & 0.33150 & 5.01508 & 0.92163 \\ \mathrm{H} & 1.34091 & 3.66567 & 1.43188 \\ \mathrm{H} & -0.42443 & 3.54658 & 1.56301 \\ \mathrm{C} & -0.97451 & 3.84436 & -1.16860 \\ \mathrm{H} & -1.02995 & 4.93772 & -1.11219 \\ \mathrm{H} & -1.86771 & 3.44028 & -0.68841 \\ \mathrm{H} & -1.00449 & 3.58589 & -2.23209 \\ \mathrm{C} & 1.50090 & 3.94462 & -1.31258 \\ \mathrm{H} & 1.35574 & 5.02472 & -1.43020 \\ \mathrm{H} & 1.55623 & 3.52022 & -2.32055 \\ \mathrm{H} & 2.46917 & 3.81220 & -0.82328 \\ \mathrm{C} & 2.14069 & 1.19388 & 0.22997 \\ \mathrm{H} & 2.80707 & 1.92660 & -0.22783 \\ \mathrm{H} & 2.12315 & 1.39365 & 1.31565 \\ \mathrm{C} & 4.08197 & -0.19302 & -0.19569 \\ \mathrm{C} & 4.68494 & 0.36483 & -1.32657\end{array}$




\begin{tabular}{|c|c|c|c|}
\hline $\mathrm{H}$ & 4.06708 & 0.81198 & -2.10166 \\
\hline $\mathrm{C}$ & 6.06716 & 0.32880 & -1.47263 \\
\hline$H$ & 6.52498 & 0.76068 & -2.3572 \\
\hline$C$ & 6.85961 & -0.28172 & -0.5014 \\
\hline $\mathrm{H}$ & 7.93730 & -0.32066 & -0.6225 \\
\hline $\mathrm{C}$ & 6.26060 & -0.84559 & 0.62048 \\
\hline $\mathrm{H}$ & 6.87053 & -1.32045 & 1.3829 \\
\hline $\mathrm{C}$ & 4.87683 & -0.79348 & 0.7826 \\
\hline $\mathrm{H}$ & 4.42863 & -1.21743 & 1.6768 \\
\hline C & 2.01819 & -1.19079 & 0.6589 \\
\hline $\mathrm{H}$ & 1.96664 & -1.00914 & 1.7453 \\
\hline 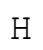 & 2.59671 & -2.10232 & 0.4954 \\
\hline C & -0.06321 & -3.30299 & 0.5198 \\
\hline $\mathrm{C}$ & 1.09946 & -4.20988 & 0.0846 \\
\hline $\mathrm{H}$ & 0.79876 & -5.25258 & 0.239 \\
\hline $\mathrm{H}$ & 2.00715 & -4.05425 & 0.6740 \\
\hline $\mathrm{H}$ & 1.35045 & -4.10511 & -0.9762 \\
\hline C & -1.33740 & -3.78369 & -0.1855 \\
\hline $\mathrm{H}$ & -1.60617 & -4.77046 & 0.2092 \\
\hline $\mathrm{H}$ & -1.18946 & -3.90205 & -1.2628 \\
\hline $\mathrm{H}$ & -2.18601 & -3.11316 & -0.04 \\
\hline $\mathrm{C}$ & -0.22134 & -3.37285 & 2.04 \\
\hline $\mathrm{H}$ & -0.41413 & -4.41162 & 2.33 \\
\hline $\mathrm{H}$ & -1.05250 & -2.76008 & 2.40 \\
\hline $\mathrm{H}$ & 0.68894 & -3.05675 & 2.5647 \\
\hline C & 0.55096 & -1.49058 & -1.8396 \\
\hline $\mathrm{H}$ & 1.62793 & -1.41761 & -2.0171 \\
\hline $\mathrm{H}$ & 0.20355 & -2.43706 & -2.2524 \\
\hline $\mathrm{C}$ & -1.52086 & -0.45720 & -2.600 \\
\hline C & -2.11441 & -1.64351 & -3.0677 \\
\hline $\mathrm{H}$ & -1.49553 & -2.48301 & -3.3670 \\
\hline C & -3.48922 & -1.73256 & -3.2325 \\
\hline $\mathrm{H}$ & -3.91581 & -2.65180 & -3.6228 \\
\hline C & -4.31710 & -0.64534 & -2.944 \\
\hline $\mathrm{H}$ & -5.38868 & -0.71346 & -3.1016 \\
\hline C & -3.74029 & 0.53641 & -2.5001 \\
\hline $\mathrm{H}$ & -4.36011 & 1.40835 & -2.3106 \\
\hline $\mathrm{C}$ & -2.35823 & 0.63348 & -2.3206 \\
\hline $\mathrm{H}$ & -1.94503 & 1.58593 & -2.0147 \\
\hline $\mathrm{C}$ & 0.50404 & 0.89202 & -2.2275 \\
\hline $\mathrm{H}$ & 0.05536 & 1.65089 & -2.873 \\
\hline $\mathrm{H}$ & 1.56552 & 0.81659 & -2.484 \\
\hline $\mathrm{C}$ & 0.04723 & 0.46605 & 3.751 \\
\hline C & 0.47170 & 0.59984 & 5.133 \\
\hline $\mathrm{H}$ & 0.28964 & -0.33374 & 5.6741 \\
\hline $\mathrm{H}$ & -0.08579 & 1.40510 & 5.6210 \\
\hline $\mathrm{H}$ & 1.53969 & 0.83317 & 5.1790 \\
\hline $\mathrm{C}$ & -3.52751 & -1.51759 & 1.548 \\
\hline $\mathrm{C}$ & -4.73148 & -2.23938 & 1.910 \\
\hline $\mathrm{H}$ & -4.53863 & -3.31658 & 1.898 \\
\hline $\mathrm{H}$ & -5.52852 & -2.01442 & 1.195 \\
\hline $\mathrm{H}$ & -5.05872 & -1.94964 & 2.913 \\
\hline $\mathrm{C}$ & -3.12881 & 2.43434 & 1.3954 \\
\hline $\mathrm{C}$ & -4.13261 & 3.40906 & 1.777 \\
\hline
\end{tabular}




$\begin{array}{llll}\mathrm{H} & -4.28098 & 3.39175 & 2.86099 \\ \mathrm{H} & -5.08292 & 3.18066 & 1.28488 \\ \mathrm{H} & -3.81244 & 4.41243 & 1.47948\end{array}$

\section{$\left[\mathrm{HCo}^{\mathrm{III}}\left(\mathrm{P}^{\mathrm{tBu}}{ }_{2} \mathrm{~N}^{\mathrm{Ph}}\right)\left(\mathrm{CH}_{3} \mathrm{CN}\right)_{3}\right]^{2+}$}

$\begin{array}{lrrr}\mathrm{Co} & -1.491997 & -0.708200 & -0.008231 \\ \mathrm{H} & -1.683229 & 0.712150 & 0.074623 \\ \mathrm{~N} & 2.305461 & -0.778797 & -0.039620 \\ \mathrm{~N} & 0.124132 & 2.183880 & 0.145195 \\ \mathrm{~N} & -1.126482 & -2.685670 & -0.122079 \\ \mathrm{P} & 0.067946 & -0.059416 & -1.479469 \\ \mathrm{P} & 0.104877 & -0.223994 & 1.489546 \\ \mathrm{C} & -0.266829 & -0.077208 & -3.339127 \\ \mathrm{C} & 1.615132 & -1.080686 & -1.276816 \\ \mathrm{H} & 1.346295 & -2.147134 & -1.368804 \\ \mathrm{H} & 2.286016 & -0.839838 & -2.103304 \\ \mathrm{C} & 4.350480 & -2.030662 & 0.595523 \\ \mathrm{H} & 3.765646 & -2.738043 & 1.176743 \\ \mathrm{C} & 5.735159 & -2.181059 & 0.530051 \\ \mathrm{H} & 6.210237 & -2.993970 & 1.070688 \\ \mathrm{C} & 6.503041 & -1.307132 & -0.232541 \\ \mathrm{H} & 7.579990 & -1.429660 & -0.284685 \\ \mathrm{C} & 5.881276 & -0.269068 & -0.925301 \\ \mathrm{H} & 6.473625 & 0.425320 & -1.513196 \\ \mathrm{C} & 4.503496 & -0.101165 & -0.846740 \\ \mathrm{H} & 4.026656 & 0.730341 & -1.360106 \\ \mathrm{C} & 3.728803 & -0.984227 & -0.088726 \\ \mathrm{C} & 1.637535 & -1.234059 & 1.154597 \\ \mathrm{H} & 2.318017 & -1.085978 & 1.996077 \\ \mathrm{H} & 1.352731 & -2.299609 & 1.132851 \\ \mathrm{C} & 0.702057 & 1.537420 & 1.296699 \\ \mathrm{H} & 0.385747 & 2.083597 & 2.188058 \\ \mathrm{H} & 1.803364 & 1.501988 & 1.280080 \\ \mathrm{C} & 0.876263 & 4.405920 & 0.951853 \\ \mathrm{H} & 1.704699 & 3.956542 & 1.492147 \\ \mathrm{C} & 0.702794 & 5.789009 & 0.989937 \\ \mathrm{H} & 1.387516 & 6.397165 & 1.573060 \\ \mathrm{C} & -0.326947 & 6.389167 & 0.272613 \\ \mathrm{H} & -0.454662 & 7.466518 & 0.299450 \\ \mathrm{C} & -1.196664 & 5.598806 & -0.478349 \\ \mathrm{H} & -2.009673 & 6.059275 & -1.031500 \\ \mathrm{C} & -1.042716 & 4.217473 & -0.502858 \\ \mathrm{H} & -1.740722 & 3.594626 & -1.056999 \\ \mathrm{C} & -0.001733 & 3.614585 & 0.209258 \\ \mathrm{C} & 0.636874 & 1.680774 & -1.111469 \\ \mathrm{H} & 1.738832 & 1.685662 & -1.150326 \\ \mathrm{H} & 0.268791 & 2.323648 & -1.913100 \\ \mathrm{C} & -0.200984 & -0.426945 & 3.343287 \\ \mathrm{C} & -1.022938 & -3.835589 & -0.193446 \\ \mathrm{H} & -1.533751 & -5.664874 & -1.078120\end{array}$




$\begin{array}{rrrr}\mathrm{H} & -1.176996 & -5.741526 & 0.664437 \\ \mathrm{H} & 0.148060 & -5.546194 & -0.508658 \\ \mathrm{~N} & -2.933424 & -0.844797 & -1.301223 \\ \mathrm{~N} & -2.905319 & -0.977526 & 1.293828 \\ \mathrm{C} & -3.851195 & -1.110988 & 1.944075 \\ \mathrm{C} & -3.892535 & -0.925838 & -1.940710 \\ \mathrm{C} & -5.022705 & -1.262552 & 2.785600 \\ \mathrm{H} & -5.550270 & -2.188387 & 2.537706 \\ \mathrm{H} & -4.723261 & -1.296809 & 3.837729 \\ \mathrm{H} & -5.699444 & -0.415066 & 2.639129 \\ \mathrm{C} & -5.082169 & -1.010129 & -2.766219 \\ \mathrm{H} & -4.813148 & -0.891757 & -3.820466 \\ \mathrm{H} & -5.568064 & -1.981178 & -2.632156 \\ \mathrm{H} & -5.785111 & -0.217986 & -2.490550 \\ \mathrm{C} & -0.600401 & -1.881325 & 3.622728 \\ \mathrm{H} & -1.513664 & -2.172260 & 3.097582 \\ \mathrm{H} & 0.193804 & -2.583445 & 3.346805 \\ \mathrm{H} & -0.774786 & -2.003247 & 4.698022 \\ \mathrm{C} & -1.298887 & 0.546879 & 3.793620 \\ \mathrm{H} & -1.550424 & 0.330519 & 4.838314 \\ \mathrm{H} & -0.966632 & 1.588826 & 3.759348 \\ \mathrm{H} & -2.211118 & 0.469106 & 3.200483 \\ \mathrm{C} & 1.082146 & -0.098109 & 4.125385 \\ \mathrm{H} & 1.488120 & 0.890405 & 3.887863 \\ \mathrm{H} & 0.837999 & -0.092970 & 5.193947 \\ \mathrm{H} & 1.871098 & -0.842227 & 3.986802 \\ \mathrm{C} & -1.382303 & 0.922789 & -3.673668 \\ \mathrm{H} & -2.283858 & 0.774425 & -3.077369 \\ \mathrm{H} & -1.059233 & 1.960103 & -3.543227 \\ \mathrm{H} & -1.649197 & 0.807552 & -4.730531 \\ \mathrm{C} & 0.999716 & 0.345954 & -4.102764 \\ \mathrm{H} & 0.739088 & 0.455232 & -5.161842 \\ \mathrm{H} & 1.395781 & 1.311669 & -3.772322 \\ \mathrm{H} & 1.799443 & -0.397759 & -4.051426 \\ \mathrm{C} & -0.656466 & -1.500385 & -3.757925 \\ \mathrm{H} & -0.845647 & -1.515761 & -4.837497 \\ \mathrm{H} & 0.147858 & -2.218280 & -3.564720 \\ \mathrm{H} & -1.559572 & -1.851449 & -3.251967 \\ & & & \end{array}$

\section{$\left[\mathrm{HCo}^{\mathrm{II}}\left(\mathrm{P}^{\mathrm{tBu}}{ }_{2} \mathrm{~N}_{2}{ }_{2}\right)\left(\mathrm{CH}_{3} \mathrm{CN}\right)_{2}\right]^{+}$}

$\begin{array}{lrrr}\text { Co } & 1.22698 & -1.34547 & 0.55203 \\ \mathrm{~N} & -2.23366 & -0.00158 & -0.25138 \\ \mathrm{~N} & 0.93562 & 1.82690 & -0.12799 \\ \mathrm{~N} & 0.19370 & -3.14407 & 0.63932 \\ \mathrm{P} & -0.17315 & -0.03486 & 1.60075 \\ \mathrm{P} & 0.23975 & -0.53907 & -1.33962 \\ \mathrm{C} & -0.21085 & 0.05702 & 3.48045 \\ \mathrm{C} & -1.93973 & -0.40481 & 1.11529 \\ \mathrm{H} & -2.11165 & -1.48370 & 1.26866 \\ \mathrm{H} & -2.61854 & 0.13145 & 1.78067\end{array}$




\begin{tabular}{|c|c|c|c|}
\hline $\mathrm{C}$ & -4.43488 & -0.27449 & -1.35435 \\
\hline 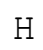 & -4.09631 & -1.16997 & -1.86636 \\
\hline C & -5.73669 & 0.17816 & -1.56459 \\
\hline $\mathrm{H}$ & -6.38303 & -0.36314 & -2.24949 \\
\hline C & -6.21345 & 1.29913 & -0.8942 \\
\hline $\mathrm{H}$ & -7.22876 & 1.64622 & -1.0576 \\
\hline & -5.37092 & 1.97526 & -0.0126 \\
\hline $\mathrm{H}$ & -5.72462 & 2.85958 & 0.5093 \\
\hline $\mathrm{C}$ & -4.06661 & 1.54071 & 0.1865 \\
\hline $\mathrm{H}$ & -3.40637 & 2.09605 & 0.847 \\
\hline $\mathrm{C}$ & -3.58252 & 0.40701 & -0.4798 \\
\hline C & -1.61322 & -0.80224 & -1.2854 \\
\hline $\mathrm{H}$ & -2.02847 & -0.48551 & -2.244 \\
\hline $\mathrm{H}$ & -1.80478 & -1.88298 & -1.17 \\
\hline C & 0.35457 & 1.32655 & -1.3602 \\
\hline $\mathrm{H}$ & 1.02804 & 1.59700 & -2.177 \\
\hline $\mathrm{H}$ & -0.63945 & 1.75081 & -1.579 \\
\hline $\mathrm{C}$ & 1.39318 & 4.00507 & -1.2237 \\
\hline $\mathrm{H}$ & 0.61492 & 3.82936 & -1.9602 \\
\hline C & 2.12970 & 5.18812 & -1.2774 \\
\hline $\mathrm{H}$ & 1.92479 & 5.90695 & -2.0655 \\
\hline C & 3.10435 & 5.45783 & -0.3232 \\
\hline $\mathrm{H}$ & 3.67208 & 6.38207 & -0.3653 \\
\hline C & 3.34921 & 4.52656 & 0.6860 \\
\hline $\mathrm{H}$ & 4.11719 & 4.71879 & 1.4298 \\
\hline $\mathrm{C}$ & 2.63334 & 3.33717 & 0.7328 \\
\hline $\mathrm{H}$ & 2.85405 & 2.59363 & 1.4946 \\
\hline $\mathrm{C}$ & 1.64245 & 3.06527 & -0.2194 \\
\hline C & 0.05651 & 1.72840 & 1.0305 \\
\hline $\mathrm{H}$ & -0.93101 & 2.18153 & 0.8379 \\
\hline $\mathrm{H}$ & 0.51809 & 2.28057 & 1.8516 \\
\hline $\mathrm{C}$ & 0.71652 & -1.06562 & -3.0871 \\
\hline $\mathrm{C}$ & -0.25856 & -4.19925 & 0.7914 \\
\hline $\mathrm{C}$ & -0.81648 & -5.52480 & 0.9964 \\
\hline $\mathrm{H}$ & -0.38809 & -5.97585 & 1.8964 \\
\hline $\mathrm{H}$ & -0.59187 & -6.16621 & 0.1393 \\
\hline $\mathrm{H}$ & -1.90219 & -5.46447 & 1.1159 \\
\hline $\mathrm{H}$ & 1.75948 & -1.55427 & 1.9215 \\
\hline $\mathrm{N}$ & 2.86518 & -2.13507 & -0.037 \\
\hline $\mathrm{C}$ & 3.92039 & -2.55553 & -0.2606 \\
\hline $\mathrm{C}$ & 5.24946 & -3.07601 & -0.5228 \\
\hline $\mathrm{H}$ & 5.70813 & -3.42238 & 0.4082 \\
\hline $\mathrm{H}$ & 5.19799 & -3.91272 & -1.225 \\
\hline $\mathrm{H}$ & 5.87837 & -2.29186 & -0.955 \\
\hline $\mathrm{C}$ & 0.47481 & -2.57730 & -3.1881 \\
\hline $\mathrm{H}$ & 1.02355 & -3.12446 & -2.4140 \\
\hline $\mathrm{H}$ & -0.58750 & -2.83137 & -3.1015 \\
\hline $\mathrm{H}$ & 0.81687 & -2.93855 & -4.1657 \\
\hline $\mathrm{C}$ & 2.21114 & -0.77635 & -3.2723 \\
\hline $\mathrm{H}$ & 2.52949 & -1.13968 & -4.2571 \\
\hline $\mathrm{H}$ & 2.43863 & 0.29433 & -3.2339 \\
\hline $\mathrm{H}$ & 2.81566 & -1.27887 & -2.5127 \\
\hline C & -0.08055 & -0.33276 & -4.1712 \\
\hline $\mathrm{H}$ & 0.03519 & 0.75472 & -4.1128 \\
\hline
\end{tabular}




$\begin{array}{rrrr}\mathrm{H} & 0.28531 & -0.64337 & -5.15766 \\ \mathrm{H} & -1.14963 & -0.56503 & -4.14152 \\ \mathrm{C} & 1.18710 & 0.43693 & 3.98086 \\ \mathrm{H} & 1.94569 & -0.26904 & 3.62954 \\ \mathrm{H} & 1.48282 & 1.44494 & 3.66902 \\ \mathrm{H} & 1.18981 & 0.42404 & 5.07728 \\ \mathrm{C} & -1.22767 & 1.09230 & 3.97783 \\ \mathrm{H} & -1.17877 & 1.14192 & 5.07203 \\ \mathrm{H} & -1.01925 & 2.09964 & 3.60145 \\ \mathrm{H} & -2.25910 & 0.83387 & 3.71835 \\ \mathrm{C} & -0.57691 & -1.33701 & 4.00422 \\ \mathrm{H} & -0.54162 & -1.33401 & 5.10024 \\ \mathrm{H} & -1.58997 & -1.63560 & 3.71165 \\ \mathrm{H} & 0.12934 & -2.09296 & 3.64496\end{array}$

\section{$\mathrm{Co}^{\mathrm{II}}(\mathrm{dmgBF})_{2}\left(\mathrm{CH}_{3} \mathrm{CN}\right)$}

\begin{tabular}{|c|c|c|c|}
\hline $\mathrm{Co}$ & 0.158582 & -0.245864 & 0.141721 \\
\hline $\mathrm{N}$ & 0.114692 & 0.115684 & 1.992956 \\
\hline 0 & 1.202589 & 0.330529 & 2.730659 \\
\hline B & 2.242498 & 1.161073 & 2.002409 \\
\hline 0 & 2.845637 & 0.409667 & 0.830611 \\
\hline $\mathrm{N}$ & 1.967355 & 0.205015 & -0.149516 \\
\hline $\mathrm{C}$ & 2.357567 & 0.208368 & -1.386895 \\
\hline $\mathrm{C}$ & 1.264970 & -0.076240 & -2.332928 \\
\hline $\mathrm{N}$ & 0.134779 & -0.335701 & -1.746173 \\
\hline 0 & -0.956208 & -0.510801 & -2.493559 \\
\hline B & -2.092581 & -1.265920 & -1.847538 \\
\hline 0 & -2.597373 & -0.589974 & -0.595692 \\
\hline $\mathrm{N}$ & -1.707477 & -0.424574 & 0.384229 \\
\hline $\mathrm{C}$ & -2.134089 & -0.240321 & 1.597900 \\
\hline $\mathrm{C}$ & -1.051981 & 0.043813 & 2.556055 \\
\hline F & 1.617614 & 2.290817 & 1.509116 \\
\hline $\mathrm{F}$ & 3.250807 & 1.370427 & 2.883061 \\
\hline $\mathrm{C}$ & 3.759360 & 0.480219 & -1.807528 \\
\hline $\mathrm{C}$ & 1.424839 & -0.027764 & -3.812244 \\
\hline $\mathrm{F}$ & -1.666721 & -2.549990 & -1.532870 \\
\hline $\mathrm{F}$ & -3.119333 & -1.221610 & -2.733556 \\
\hline $\mathrm{C}$ & -3.575148 & -0.269238 & 1.970458 \\
\hline $\mathrm{C}$ & -1.275321 & 0.237121 & 4.015029 \\
\hline $\mathrm{H}$ & -1.459360 & -0.722315 & 4.514437 \\
\hline $\mathrm{H}$ & -0.387821 & 0.689149 & 4.460175 \\
\hline $\mathrm{H}$ & -2.143717 & 0.877660 & 4.197176 \\
\hline $\mathrm{H}$ & -3.717847 & -0.721212 & 2.955995 \\
\hline $\mathrm{H}$ & -3.988033 & 0.746915 & 2.006196 \\
\hline $\mathrm{H}$ & -4.136597 & -0.829918 & 1.221168 \\
\hline $\mathrm{H}$ & 1.352936 & 1.004592 & -4.177479 \\
\hline $\mathrm{H}$ & 0.628407 & -0.603938 & -4.286378 \\
\hline $\mathrm{H}$ & 2.398867 & -0.421243 & -4.116043 \\
\hline $\mathrm{H}$ & 3.786810 & 1.167161 & -2.658996 \\
\hline $\mathrm{H}$ & 4.264686 & -0.445248 & -2.110726 \\
\hline $\mathrm{H}$ & 4.310709 & 0.912475 & -0.971301 \\
\hline I & 0.926776 & -5.296594 & -0.582387 \\
\hline
\end{tabular}




$\begin{array}{lrrr}\mathrm{H} & -0.238586 & -5.352493 & 0.764032 \\ \mathrm{H} & -0.768323 & -4.796636 & -0.850066 \\ \mathrm{C} & 0.059070 & -4.805002 & -0.134305 \\ \mathrm{C} & 0.366217 & -3.426313 & 0.188794 \\ \mathrm{~N} & 0.566408 & -2.309646 & 0.408442\end{array}$

\section{$\mathrm{HCo}^{\mathrm{III}}\left(\mathrm{dmgBF}_{2}\right)_{2}\left(\mathrm{CH}_{3} \mathrm{CN}\right)$}

\begin{tabular}{|c|c|c|c|}
\hline $\mathrm{CO}$ & 0.118825 & -0.199043 & 0.109538 \\
\hline $\mathrm{N}$ & 0.090912 & 0.102760 & 1.958709 \\
\hline O & 1.180272 & 0.260949 & 2.703022 \\
\hline B & 2.287041 & 1.056450 & 2.036801 \\
\hline 0 & 2.817720 & 0.340072 & 0.809293 \\
\hline $\mathrm{N}$ & 1.930258 & 0.191681 & -0.168522 \\
\hline $\mathrm{C}$ & 2.325203 & 0.193869 & -1.405456 \\
\hline C & 1.235917 & -0.061147 & -2.352238 \\
\hline $\mathrm{N}$ & 0.098416 & -0.289948 & -1.767281 \\
\hline O & -0.991549 & -0.447019 & -2.513822 \\
\hline B & -2.130876 & -1.203814 & -1.877652 \\
\hline O & -2.625518 & -0.526087 & -0.624045 \\
\hline $\mathrm{N}$ & -1.735664 & -0.378642 & 0.353936 \\
\hline $\mathrm{C}$ & -2.158157 & -0.225246 & 1.573118 \\
\hline $\mathrm{C}$ & -1.074554 & 0.029560 & 2.526449 \\
\hline $\mathrm{F}$ & 1.789776 & 2.280925 & 1.657997 \\
\hline $\mathrm{F}$ & 3.312764 & 1.081495 & 2.924759 \\
\hline C & 3.737281 & 0.431911 & -1.814224 \\
\hline C & 1.397355 & -0.019723 & -3.832061 \\
\hline $\mathrm{F}$ & -1.710809 & -2.490405 & -1.568281 \\
\hline $\mathrm{F}$ & -3.157351 & -1.149773 & -2.762919 \\
\hline $\mathrm{C}$ & -3.599305 & -0.261393 & 1.946535 \\
\hline $\mathrm{C}$ & -1.283238 & 0.189354 & 3.992273 \\
\hline $\mathrm{H}$ & -1.457919 & -0.781042 & 4.473365 \\
\hline $\mathrm{H}$ & -0.394035 & 0.635915 & 4.439299 \\
\hline $\mathrm{H}$ & -2.152348 & 0.822225 & 4.195781 \\
\hline $\mathrm{H}$ & -3.740026 & -0.721098 & 2.928615 \\
\hline $\mathrm{H}$ & -4.014742 & 0.753367 & 1.988673 \\
\hline $\mathrm{H}$ & -4.160349 & -0.818472 & 1.194256 \\
\hline $\mathrm{H}$ & 1.338467 & 1.012380 & -4.200023 \\
\hline $\mathrm{H}$ & 0.594394 & -0.587104 & -4.305709 \\
\hline $\mathrm{H}$ & 2.366126 & -0.427217 & -4.133836 \\
\hline $\mathrm{H}$ & 3.786059 & 1.110113 & -2.671533 \\
\hline $\mathrm{H}$ & 4.227098 & -0.506198 & -2.103460 \\
\hline $\mathrm{H}$ & 4.290303 & 0.861066 & -0.977617 \\
\hline $\mathrm{H}$ & 1.111568 & -5.177826 & -0.418830 \\
\hline $\mathrm{H}$ & -0.053999 & -5.234034 & 0.927661 \\
\hline $\mathrm{H}$ & -0.621698 & -4.872439 & -0.727525 \\
\hline $\mathrm{C}$ & 0.196608 & -4.737493 & -0.013576 \\
\hline C & 0.367340 & -3.314362 & 0.193733 \\
\hline $\mathrm{N}$ & 0.452963 & -2.170939 & 0.31601 \\
\hline $\mathrm{H}$ & -0.142096 & 1.193196 & -0.0578 \\
\hline
\end{tabular}

